\title{
Planetary physics research at the Facility for Antiprotons and Ion Research using intense ion beams
}

\author{
N. A. Tahir ${ }^{1, a}$, A. Shutov ${ }^{2}$, P. Neumayer ${ }^{1}$, V. Bagnoud ${ }^{1}$, A. R. Piriz ${ }^{3}$, S. A. Piriz ${ }^{3}$, C. Deutsch ${ }^{4}$ \\ ${ }_{1}^{1}$ GSI Helmholtzzentrum für Schwerionenforschung, Planckstraße 1, 64291 Darmstadt, Germany \\ ${ }^{2}$ Institute of Problems of Chemical Physics, Russian Academy of Sciences, Institutskii pr. 18, Chernogolovka, Russia 142432 \\ 3 E.T.S.I.Industriales, Universidad de Castilla-La Mancha, 13071 Ciudad Real, Spain \\ ${ }^{4}$ Laboratoire de Physik des Gaz et des Plasmas, Universite Paris-Sud, 91405 Orsay, France
}

Received: 14 January 2022 / Accepted: 11 February 2022

(C) The Author(s) 2022

\begin{abstract}
Intense particle beams offer a new efficient driver to produce extended samples of high energy density (HED) matter with extreme physical conditions that are expected to exist in the planetary interiors. In this paper, we present two-dimensional hydrodynamic implosion simulations of a multi-layered cylindrical target that is driven by an intense uranium beam. The target is comprised of a sample material (which is water in the present case) that is enclosed in a cylindrical tungsten shell. This scheme is named LAPLAS that stands for Laboratory Planetary Science, and it leads to a low entropy compression. This means that the water sample is compressed to super-solid densities, ultra-high pressures, but relatively low temperatures. Such exotic conditions are predicted to exist in the cores of water-rich solar, as well as extrasolar planets. The beam parameters are chosen to match the characteristics of the particle beam that will be delivered by the heavy ion synchrotron, SIS100, at the Facility for Antiprotons and Ion Research (FAIR), at Darmstadt. It is to be noted that the LAPLAS scheme is an important part of the HED physics program at FAIR, which is named HEDP@FAIR. The simulations predict that the LAPLAS experiments will produce a wealth of information on the Equation-of-State properties of the exotic matter that forms the planetary cores. This information can be very helpful in understanding the formation, evolution and the final structure of the planets.
\end{abstract}

\section{Introduction}

Planetary physics [1-16] belongs to the field of research named High Energy Density (HED) physics [17-19] that spans over vast areas of basic and applied sciences. Progress in high pressure physics during the past decades has made it possible to produce samples of HED matter with extreme physical conditions in the laboratory. Static as well as dynamic techniques have been used for this purpose. In the former type of experiments, HED states are induced in the sample at room temperature, as it is squeezed in a closed vessel or between anvils. Diamond Anvil Cell (DAC) is a typical example of a static scheme [20,21]. It is also possible to heat the sample with a laser during compression to achieve a higher temperature, for example, see [22], which reports a temperature of around $3500 \mathrm{~K}$. The time scale of such experiments is from hours to days. In a dynamic scheme, on the other hand, HED matter is generated by the application of shocks. Shock compression of matter leads to irreversible heating that generates states of high entropy and high pressure in the sample. Most of the experimental studies on Equation of State (EOS) of HED matter have been done using such techniques. The shocks are generated by powerful generators including high power chemical explosives, light gas guns, Z-pinches [23-26] and powerful lasers [27-29]. Much higher pressures in the Gbar range have been generated using underground nuclear explosions [30].

Significant technological developments in the field of accelerators have led to the availability of high intensity particle beams [3134]. Theoretical studies on beam-matter heating have shown that such beams are an additional, very efficient tool to produce samples of HED matter in the laboratory [35-55]. It is interesting to note that using an ion beam, HED states can be induced in matter using two very different schemes. One scheme is based on isochoric and uniform heating of matter by the beam, while the other employs shock compression of the material. This flexibility is not provided by any other generator. An experiment based on the former scheme named HIHEX (Heavy Ion Heating and Expansion) has been designed with the help of detailed numerical simulations to study the EOS properties of HED matter [43,56-58]. The second scheme is employed to propose another experiment named, LAPLAS, that stand for Laboratory Planetary Science and that uses a multiple shock reflection technique that leads to a low-entropy compression of a sample material which is enclosed in a cylindrical high- $Z$ shell. This multi-layered target is driven by an ion beam that has an annular focal spot. The sample material is imploded to super-solid densities, ultra-high pressures, while the temperature remains relatively low. These are the typical exotic conditions expected to exist in the planetary cores. It is to be noted that the

\footnotetext{
a e-mail: n.tahir@gsi.de (corresponding author)
} 
HIHEX and the LAPLAS experimental schemes are the major part of the HED physics research program at FAIR, which is named HEDP@FAIR [59].

In the present paper, we present numerical simulations of implosion of the LAPLAS scheme. This work underlines the advantages of the LAPLAS scheme compared to other HED drivers; one of the drawbacks is that the sample of interest is hidden inside the heavy material shell used for compression. State-of-the art radiographic techniques, which are often based on X-rays, cannot probe efficiently such a target from the side because of the shielding effect of the shell. In addition, the long time scales of the compression phase are not favorable for building in view holes, as they would fill up quickly during the plasma expansion. For this reason, the most appropriate way to probe the sample is to do an on-axis radiography, where a hard X-ray source is located on axis close to the target. By doing so, it is advantageous to optimize the target longitudinal length in order to find the best trade-off between a thin amount of compressed material that enables releasing the requirement on the X-ray source and the necessity to keep an aspect ratio where one-dimensional effects still dominate. In this paper, we present a two-dimensional study with emphasis optimizing the LAPLAS target length for this purpose.

In Sect. 2, we describe the LAPLAS scheme, while in Sect. 3, the beam and the target parameters are given. Optimization of the target dimensions in relation to the facilitation of diagnostics is discussed in Sect. 4, whereas the simulation results are presented in Sect. 5. Conclusions drawn from this study are noted in Sect. 6.

\section{Description of the LAPLAS scheme}

Beam-target geometry of the LAPLAS experiment is shown in Fig. 1. The target is a multi-layered cylinder made of a sample material that is enclosed in a heavy shell of a high- $\mathrm{Z}$ material. One face of the target is irradiated with an intense heavy ion beam that has an annular (ring-shaped) focal spot. The annular focal spot can be generated using an rf-wobbler that rotates the beam with very high frequency. Such a system is designed for this experiment within the framework of the HEDP@FAIR collaboration. Detailed analysis of the energy deposition symmetry issues related to a wobbler has been analyzed and reported in [60], while design of a prototype wobbler system has been reported in [61].

It is assumed that the inner radius of the annulus is larger than the radius of the sample material, which is a necessary condition to avoid strong direct heating of the sample by the ion beam (see Fig. 2, which is the cross-sectional view of the target shown in Fig. 1). Moreover, it is considered that the outer radius of the focal spot ring is smaller than the outer radius of the surrounding high- $Z$ shell. It is seen in Fig. 2, that a layer of cold material from the high-Z shell known as "pusher" or "payload" is created between the sample material and the beam-heated region. The payload plays an important role in placing the compression on the desired adiabat.

Fig. 1 Beam-target set up of LAPLAS scheme

Fig. 2 Cross-sectional view of the target shown in Fig. 1
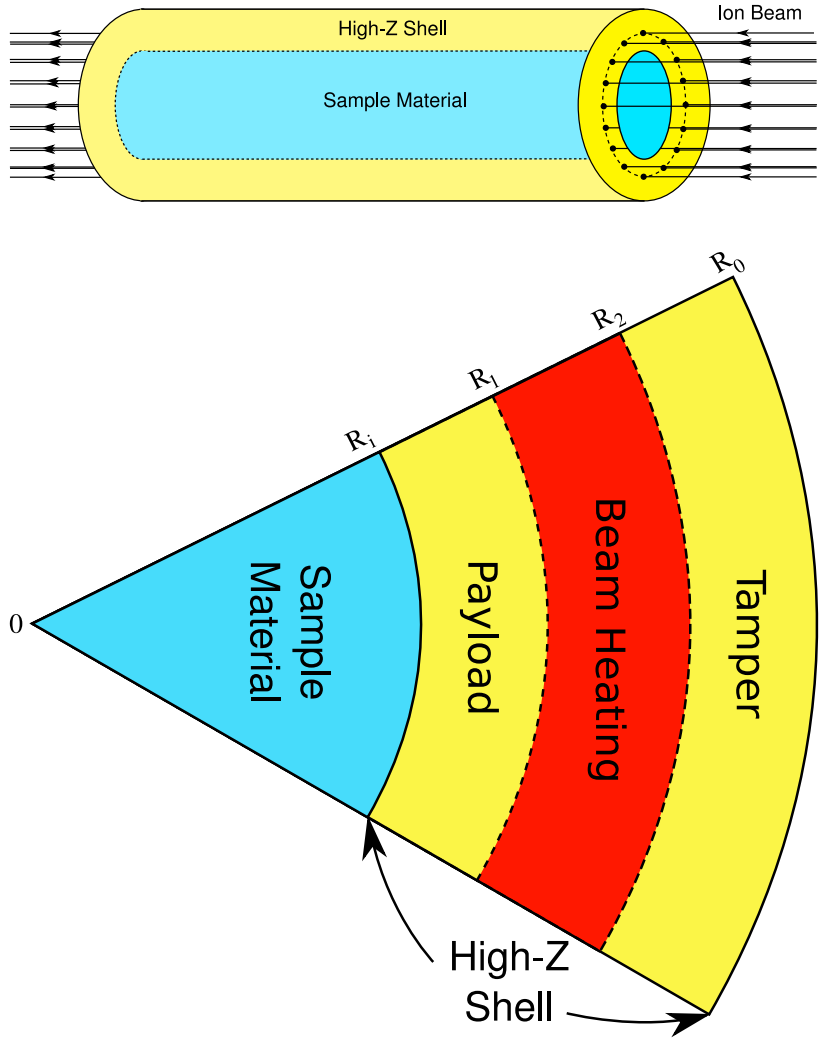
It is also seen that a cold shell around the beam-heated zone remains as a tamper that confines the implosion for a longer time. The target length is assumed to be less than the range of the driver ions so that the energy deposition in the longitudinal direction is uniform. The pressure in the beam heated region increases substantially that launches a shock wave inwards, along the radial direction. The shock wave enters the pusher, is subsequently transmitted into the sample, and then reflected at the cylinder axis. The reflected shock moves outwards along the radial direction and is re-reflected at the sample-shell boundary. This process is repeated a few times, while the boundary continues to move inwards, thereby compressing the sample slowly. The optimum sample physical conditions are achieved when the inward motion of the payload is stopped by the high pressure in the compressed sample. This scheme generates a low-entropy compression of the sample material that leads to the exotic physical conditions that are expected to exist in the planetary cores.

\section{Beam and target parameters used in the study}

In the present study, the sample is frozen water (ice), whereas the surrounding shell is made of tungsten. It is worth noting that the quasi-isentropic implosion of LAPLAS depends on the high density of the payload. On the other hand, the hydrodynamic stability of the imploding payload (see Fig. 2) relies on the mechanical properties of the payload material; the most suitable materials are those with the highest yield strength. Tungsten is one of the materials that better satisfy these two requirements. Recent analysis has shown $[62,63]$ that the implosion can be sufficiently stable, provided that the symmetry below $2 \%$ in the driving pressure is achieved. Such a symmetry level is expected to be achievable with the wobbler system that will rotate the beam with a frequency of $1 \mathrm{GHz}[60]$.

We consider a uranium beam with a particle energy of $1.5 \mathrm{GeV} / \mathrm{u}$. The beam intensity is $5 \times 10^{11}$ ions/bunch, which is the maximum value delivered by the SIS100. Also the bunch length is $75 \mathrm{~ns}$. The focal spot geometry is ring shaped and the precise parameters used in different cases are noted in Table 1.

It is seen in Table 1 that two values of ice radius, namely $R_{i}=0.2 \mathrm{~mm}$ and $0.3 \mathrm{~mm}$, respectively, are considered. For the former case, two different values of the inner ring radius, $R_{1}=0.3 \mathrm{~mm}$ and $0.4 \mathrm{~mm}$, respectively, are used. This allows a reasonable mass of tungsten payload around the sample material. For the same reason, in case of $R_{i}=0.3 \mathrm{~mm}$, we use $R_{1}=0.4 \mathrm{~mm}$ and $0.5 \mathrm{~mm}$, respectively. In all the above cases, the width of the ring is assumed to be $1 \mathrm{~mm}$. It is important to note that the transverse intensity distribution in the focal spot is a Gaussian. Therefore, due to the wings of the Gaussian distribution, it may be difficult to generate a completely hollow focal spot that will lead to a certain level of energy deposition in the sample and the pusher as well. For the simplicity of the calculations, we consider a spatial power deposition profile as shown in Fig. 3, which is comprised of a constant power step with an amplitude A (which lies in the sample and the payload regions), that is followed by a parabolic power profile. We consider different values of the amplitude, A, varying from 10 to $40 \%$ to see the effect of the sample preheat on compression.

Table 1 Target and focal spot parameters

\begin{tabular}{lllll}
\hline Case no & $R_{i}(\mathrm{~mm})$ & $R_{1}(\mathrm{~mm})$ & $R_{2}(\mathrm{~mm})$ & $R_{o}(\mathrm{~mm})$ \\
\hline 1 & 0.2 & 0.4 & 1.4 & 3.0 \\
2 & 0.2 & 0.3 & 1.3 & 3.0 \\
3 & 0.3 & 0.5 & 1.5 & 3.0 \\
4 & 0.3 & 0.4 & 1.4 & 3.0 \\
\hline
\end{tabular}

Fig. 3 Spatial power deposition profile

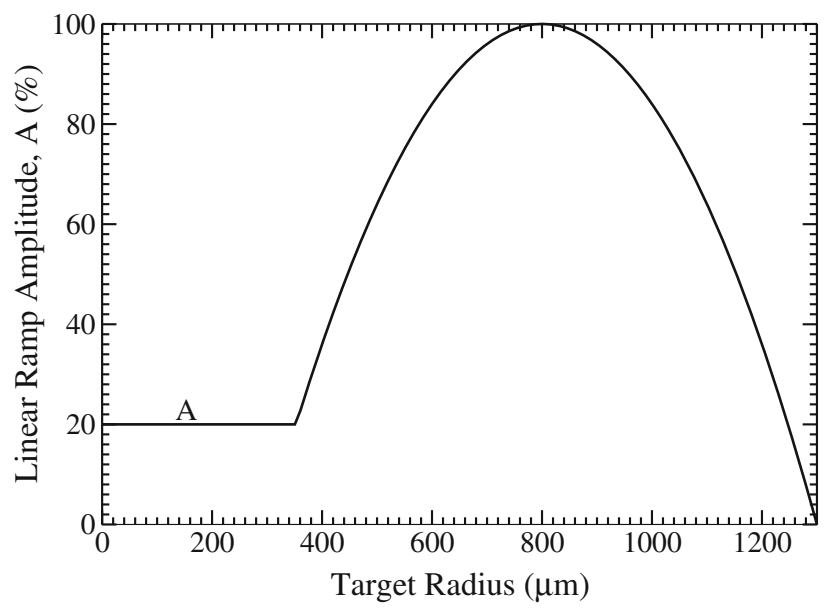


Previously [64], we reported extensive 1D hydrodynamic simulation studies of compression of the LAPLAS target along the radial direction. A wide range of the beam and the target parameters were considered. This study showed that a wide region of the water phase diagram can be accessed in such experiments. However, it is necessary to determine the minimum target length that still allows for the validity of 1D approximation. This is important because a shorter target is more suitable for the efficiency and accuracy of the diagnostics. For this purpose, we have performed full 2D simulations considering the four different cases noted in Table 1, using four different values of target length, including 1, 2, 3 and $4 \mathrm{~mm}$, respectively. Moreover, we have considered different values of the amplitude, $A=0.1,0.2,0.3$ and 0.4 , respectively (see Fig. 3).

\section{Optimum target parameters for diagnostic facilitation}

In the LAPLAS scheme, the sample material is surrounded by a heavy material cylindrical shell. Along the heavy ion beam direction, the access to the sample material is free, though. Therefore, it is advantageous to use this direction for the measurements to facilitate the access to the sample material.

Surface measurements using optical probing by, for example, pyrometry or VISAR are most likely unable to deliver a full picture of the obtained state of matter. By contrast, penetrating radiation such as hard X-rays can be employed to complement the previous diagnostics and yield information from within the volume of the compressed sample. Indeed, a variety of measurement techniques based on intense X-ray sources have been developed in the last decades as diagnostic in HED-science experiments, facilitated by the ever growing experimental capabilities offered by optical and X-ray short pulse lasers.

Adapting laser-driven X-ray probing to the LAPLAS scheme, one has to consider several aspects. First of all, X-ray based absorption methods that are adapted to determine the volume and areal density of the compressed material are not currently adapted to very thick samples. Second, the ion-beam parameters available at FAIR for LAPLAS dictate the size and volume of the compressible material. It results that the objects to radiograph have a radius below $100 \mu \mathrm{m}$, which limits the solid angle accessible for diagnosing the target, especially when using long targets.

As an example, laser-driven hard X-ray sources based on the emission excited in solid targets by the hot electrons produced in relativistic laser-matter interaction emit photons reaching $\mathrm{MeV}$ energies. However, the emitted bremsstrahlung spectrum falls off rapidly at photon energies beyond several $10 \mathrm{~s}$ of $\mathrm{keV}$ [65]. Likewise, the conversion efficiency into $K$-alpha line emission was found to decrease by a factor 4 when going from $\mathrm{Ag}(22 \mathrm{keV})$ to $\mathrm{Pb}(75 \mathrm{keV})$ [66]. Similarly, betatron radiation produced from ultra-intense short-pulse lasers in a gas jet has a high diagnostic potential due to the small source size, high directionality, and short duration. However, even at laser intensities greater than $10^{19} \mathrm{~W} / \mathrm{cm}^{2}$ most of the betatron radiation is concentrated in the spectral range of 10-30 keV [67]. Given the manifold compression reached in the LAPLAS scheme, reducing the target length as investigated in this study would significantly increase transmission at these lower photon energies and thereby help to relax the requirements on the $\mathrm{X}$-ray source.

To give an example, for the case studied in this paper with water as the sample material, the mass attenuation coefficient of water at $22 \mathrm{keV}$ photon energy (Ag K-alpha) is $0.66 \mathrm{~cm}^{2} / \mathrm{g}$ [68]. At a target length of $2 \mathrm{~mm}$, the X-ray transmission at normal density would be as high as $88 \%$, dropping to $48 \%$ at the highest density of $5.5 \mathrm{~g} / \mathrm{cm}^{3}$ reached at peak compression. This transmission is ideal for optimum signal-to-noise and density contrast in a transmission measurement to assess the overall areal density of the compressed sample.

Another important consequence of a reduced target length is the geometric access to the compressed sample. This is crucial when performing X-ray diffraction measurements to assess the crystallographic structure. At the thermodynamic parameters reached in the water sample as predicted in this paper, the sample should be in the high-density ice X-phase with a body-centered-cubic oxygen lattice, and at higher densities and temperature in the super-ionic phase, predicted to be in a face-centered-cubic lattice of oxygen atoms [69]. Based on recent diffraction measurements on laser-compressed water samples [70], and assuming a $22 \mathrm{keV}$ probe line, the 110 -reflection from the ice $X$ at $3.8 \mathrm{~g} / \mathrm{cm}^{3}$ would appear at $2 \Theta=18.3^{\circ}$, while the 111 -reflection from super-ionic water at $4.5 \mathrm{~g} / \mathrm{cm}^{3}$ would be at $2 \Theta=18.9^{\circ}$. Given these diffraction angles, even for the implosions with smaller initial sample radius (cases 1 and 2) would still allow to observe diffraction from up to $0.5 \mathrm{~mm}$ into the sample at peak compression.

\section{Numerical simulation results}

In this section, we present 2D hydrodynamic simulation results obtained employing a 2D hydrodynamic computer code, BIG2 [71], considering the four different beam-target cases noted in Table 1. This code is based on a Godunov type numerical algorithm [72] and considers an Eulerian numerical scheme that uses curvilinear rectangular moving grid. The code can treat multi-layered targets comprised of different materials and can handle complicated target geometries. The ion beam energy deposition in the material is calculated assuming a cold stopping model described in [73]. Since the target temperature in these calculations is below 10 eV, the level of ionization is negligible, and thus, the cold stopping model is a valid approximation. A semi-empirical equation-of-state (EOS) model $[74,75]$ is used for tungsten, while for water, the SESAME data [76] is considered. The mechanical (elastic-plastic) 


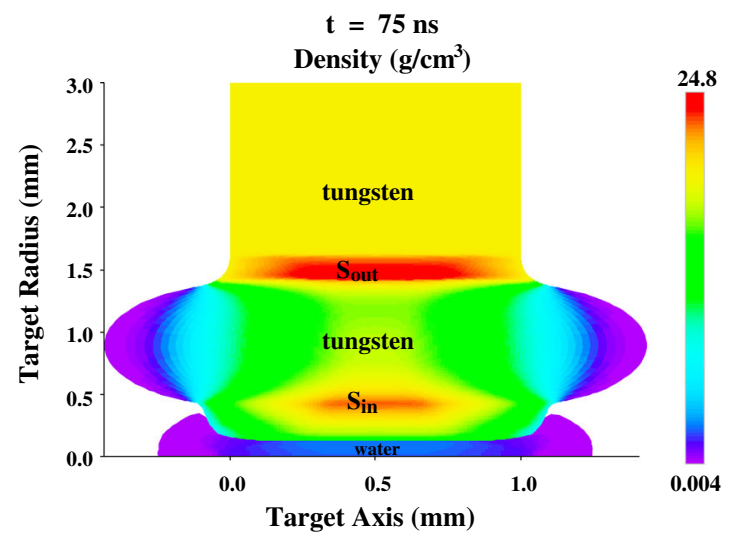

(a)

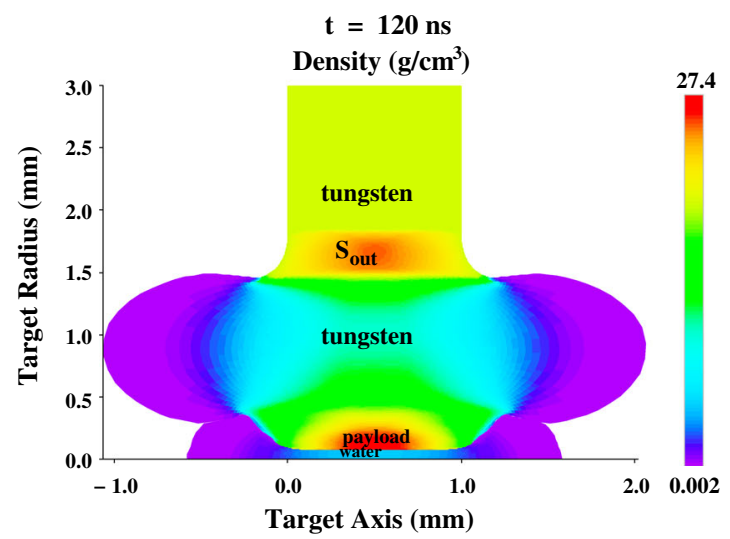

(c)

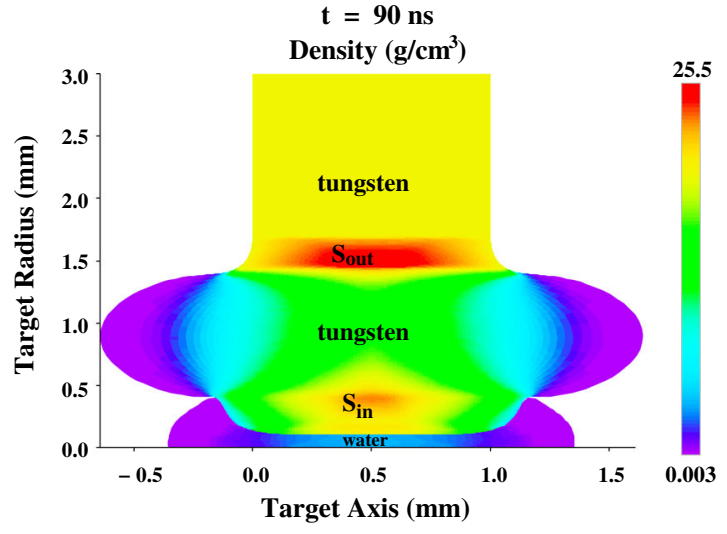

(b)

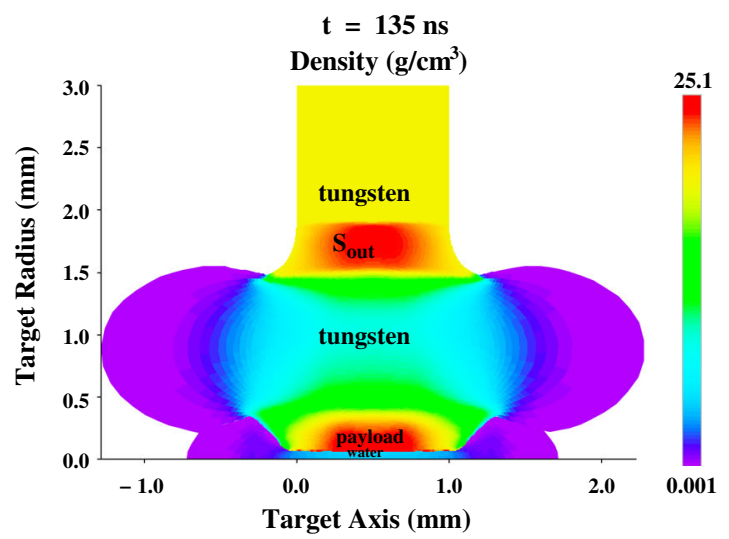

(d)

Fig. 4 Target density distribution at different times generated by BIG2 code, target length $=1 \mathrm{~mm}$, intensity $=5 \times 10^{11}$ uranium ions per bunch, particle energy $=1.5 \mathrm{GeV} / \mathrm{u}$, bunch length $=75 \mathrm{~ns}$, ring shaped focal spot, inner ring radius, $R_{1}=0.4 \mathrm{~mm}$, outer ring radius, $R_{2}=1.4 \mathrm{~mm}$, sample radius, $R_{i}=0.2 \mathrm{~mm}$, outer cylinder radius, $R_{O}=3 \mathrm{~mm}$ (see Fig. 2), foot amplitude, $A=0.2$ (see Fig. 3); $\mathbf{a}$ at $t=75 \mathrm{~ns}, \mathbf{b}$ at $t=90 \mathrm{~ns}$, $\mathbf{c}$ at $t=120 \mathrm{~ns}$ and $\mathbf{d}$ at $t=135 \mathrm{~ns}$

properties of solid materials are treated using the nonlinear Prandtl-Reuss model with the von Mises yield criterion [77]. Further details about the code can be found in $[64,71]$.

\subsection{Case 1}

Here we present simulation results considering Case 1 noted in Table I, which means a sample radius, $R_{i}=0.2 \mathrm{~mm}$, an inner ring radius, $R_{1}=0.4 \mathrm{~mm}$ and an outer ring radius, $R_{2}=1.4 \mathrm{~mm}$. We have used different values of the amplitude, $A$ (see Fig. 3 ), including $0.1,0.2,0.3$ and 0.4 , respectively. However, in this paper we report the results corresponding to $A=0.2$ only.

\subsubsection{Target length $1 \mathrm{~mm}$}

First, we discuss the case with cylinder length, $L=1 \mathrm{~mm}$. In Fig. 4, we plot the target density distribution produced by the BIG2 code at different times. Figure. $4 \mathrm{a}$ is plotted at $t=75 \mathrm{~ns}$, the time when the bunch has just delivered its total energy. The beam enters the target from the right side, deposits a fraction of its energy in the target material and emerges from the left side. It is seen that two shocks, $\mathrm{S}_{\text {out }}$ and $\mathrm{S}_{\text {in }}$, have developed, above and below the high pressure beam heated tungsten region. The former shock moves radially outwards, whereas the latter propagates inwards. It is also seen that the inmoving shock is relatively weaker because the pusher is preheated by the wings of the Gaussian distribution represented by $A=0.2$, in the present case. The figure also shows rarefaction waves penetrating into the target from both faces and significant material expansion has taken place.

Figure $4 \mathrm{~b}$ is plotted at $t=90 \mathrm{~ns}$, which shows that the rarefaction waves have further penetrated into the target from both sides and the lateral material expansion has increased further. It is also to be noted that due to the preheating, the water sample also expands that affects the lateral uniformity and quality of the final compression.

Figure $4 \mathrm{c}$ shows that at $t=120 \mathrm{~ns}$, a small payload shell with a density of about $27 \mathrm{~g} / \mathrm{cm}^{3}$ has been formed around the water. The maximum water density at the center of the cylinder axis is about $4.5 \mathrm{~g} / \mathrm{cm}^{3}$, while the pressure is $4.7 \mathrm{Mbar}$. 
Table 2 Optimum physical conditions in compressed water obtained using 1D hydrodynamic model, bunch intensity $=5 \times 10^{11}$ uranium ions, bunch length $=75 \mathrm{~ns}$, particle energy $=1.5 \mathrm{GeV} / \mathrm{u}$, sample radius, $R_{i}=0.2 \mathrm{~mm}$, annular focal spot with inner ring radius, $R_{1}=0.4 \mathrm{~mm}$, outer ring radius, $R_{2}=1.4 \mathrm{~mm}[64]$

\begin{tabular}{llllll}
\hline No & $A(\%)$ & $\rho\left(\mathrm{g} / \mathrm{cm}^{3}\right)$ & $P(\mathrm{Mbar})$ & $T(\mathrm{~K})$ & $t_{\text {impl }}(\mathrm{ns})$ \\
\hline 1 & 10 & 6.05 & 9.7 & $6.6 \times 10^{3}$ \\
2 & 20 & 5.52 & 8.03 & $9 \times 10^{3}$ \\
3 & 30 & 5.2 & 7.0 & $1.16 \times 10^{4}$ \\
4 & 40 & 4.9 & 6.2 & $1.35 \times 10^{4}$ \\
\hline
\end{tabular}

Fig. 5 Density, temperature, and material velocity along the cylinder axis at $t=135 \mathrm{~ns}$, $L=1 \mathrm{~mm}$

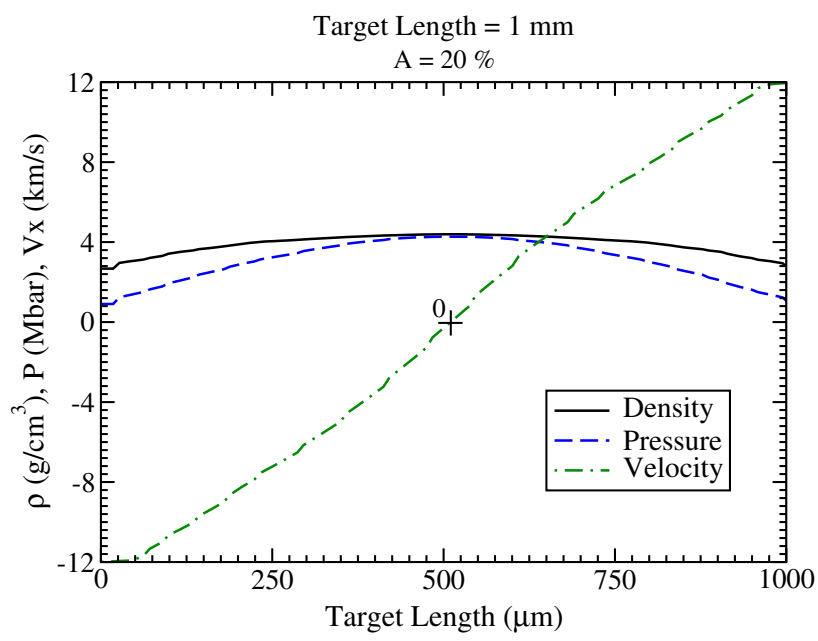

Figure $4 \mathrm{~d}$ is plotted at $t=135 \mathrm{~ns}$, which is the time of maximum compression according to the $1 \mathrm{D}$ simulations noted in Table 2 . It is seen that the density of the payload is slightly reduced compared to that in Fig. $3 \mathrm{c}$, due to the lateral expansion. The maximum water density at this time is $4.1 \mathrm{~g} / \mathrm{cm}^{3}$, and the maximum pressure is $3.7 \mathrm{Mbar}$ at the center of the axis. However, Table 2 shows that the 1D simulations predict a maximum density is $5.5 \mathrm{~g} / \mathrm{cm}^{3}$ and a maximum pressure of around $8 \mathrm{Mbar}$ in water in case of $A=20 \%$. This comparison shows that because of the 2D effects, $1 \mathrm{~mm}$ length of the LAPLAS target is not enough to provide the optimum physical conditions in the sample.

To further illustrate this point, we plot in Fig. 5, the density, the pressure and the material velocity along the target axis at $t=135 \mathrm{~ns}$, the time of maximum compression. We note that in this figure we exclude the low-density expanded region and only consider the inner $1 \mathrm{~mm}$ zone, which is the initial target length. It is seen that the density and the pressure are not uniform along the axis, but have a maximum value at the middle of the axis that symmetrically decrease on either side. Also the material velocity is zero only at the middle point, while it increases rapidly to the left and to the right of this central point. This shows that the entire sample material is non-stationary and is expanding with high velocity. These conditions are not very favorable for accurate measurements. Therefore, a 1-mm-long target is not a viable option.

\subsubsection{Target length $2 \mathrm{~mm}$}

In this sub-section, we present the results obtained using a cylinder with length, $L=2 \mathrm{~mm}$. In Fig. 6 , we present the target density distribution provided by the BIG2 code at different times. Figure 6a shows that two shocks developed at $t=75 \mathrm{~ns}$ have similar strengths as the corresponding shocks in the previous case, but cover a wider length due to the increased length of the target. It is also interesting to note that the region occupied by the expanded material on either side of the target is the same as in the case with $L=1 \mathrm{~mm}$. However, due to the larger length of the cylinder, the rarefaction waves have not yet penetrated deep enough to affect the entire sample material.

Figure $6 \mathrm{~b}$ is plotted at $t=90 \mathrm{~ns}$, that shows that the strength of the shocks as well as the region occupied by the expanded material is the same as in Fig. 4b, but the lengths of the shock fronts and that of the compressed sample are larger in the present case.

Figure $6 \mathrm{c}$ is plotted at $t=120 \mathrm{~ns}$, which shows that the density of the payload is about $32 \mathrm{~g} / \mathrm{cm}^{3}$ that is higher compared to $27.4 \mathrm{~g} / \mathrm{cm}^{3}$ in Fig. 4c. Moreover, the length of the payload shell is larger in the present case.

Similar behavior is seen in Fig. 6d, which is plotted at $t=135 \mathrm{~ns}$, the time of maximum compression.

In Fig. 7, we plot the density, the pressure and the material velocity along the target axis at $t=135 \mathrm{~ns}$, the time of optimum compression. In this figure, we exclude the expanded part of the target and include only the inner 2 mm cylinder length, which is the 


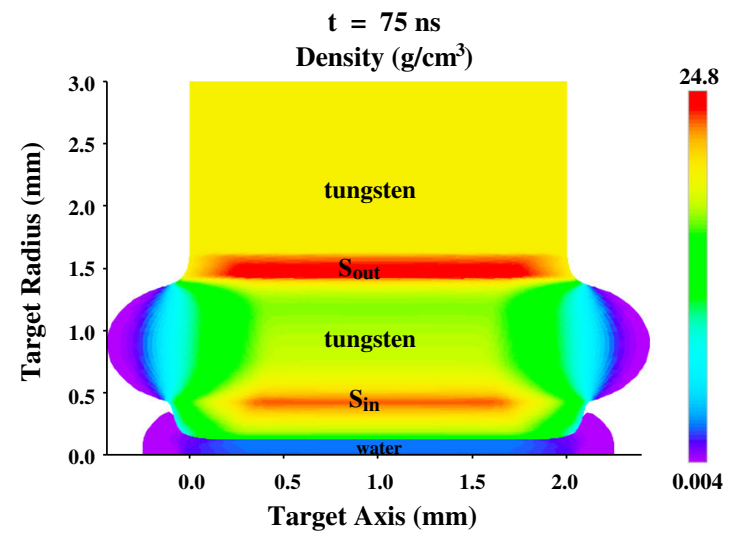

(a)

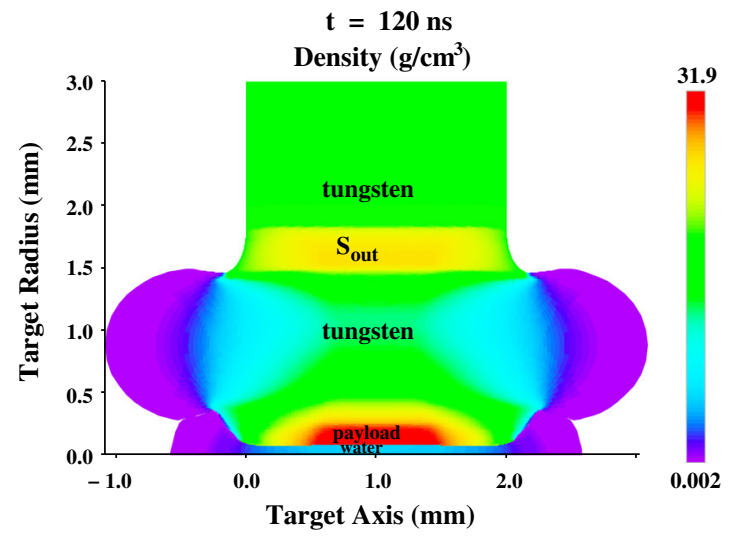

(c)

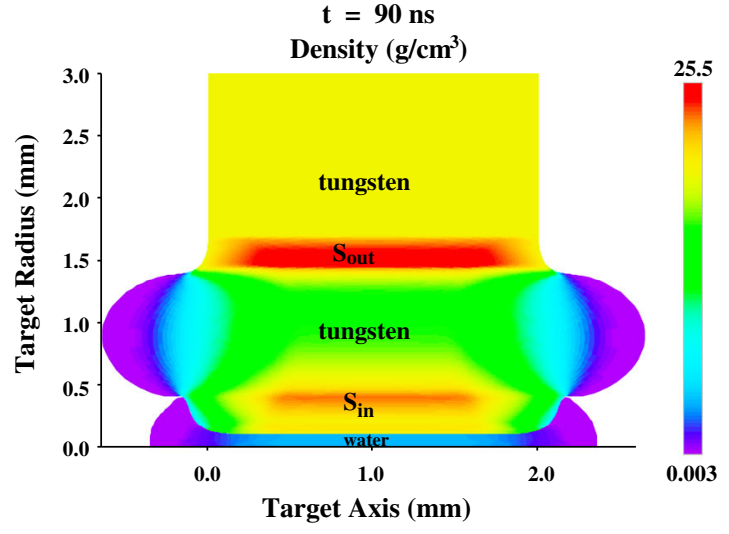

(b)

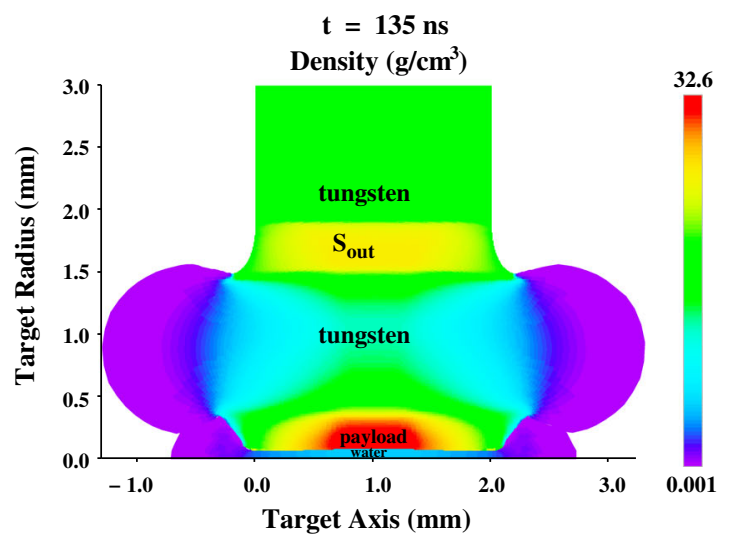

(d)

Fig. 6 Target density distribution at different times generated by BIG2 code, target length $=2 \mathrm{~mm}$, intensity $=5 \times 10^{11}$ uranium ions per bunch, particle energy $=1.5 \mathrm{GeV} / \mathrm{u}$, bunch length $=75 \mathrm{~ns}$, ring shaped focal spot, inner ring radius, $R_{1}=0.4 \mathrm{~mm}$, outer ring radius, $R_{2}=1.4 \mathrm{~mm}$, sample radius, $R_{i}=0.2 \mathrm{~mm}$, outer cylinder radius, $R_{O}=3 \mathrm{~mm}$ (see Fig. 2), foot amplitude, $A=0.2$ (see Fig. 3); $\mathbf{a}$ at $t=75 \mathrm{~ns}$, b at $t=90 \mathrm{~ns}, \mathbf{c}$ at $t=120 \mathrm{~ns}$ and $\mathbf{d}$ at $t=135 \mathrm{~ns}$

Fig. 7 Density, pressure and material velocity along cylinder axis at $t=135 \mathrm{~ns}, L=2 \mathrm{~mm}$

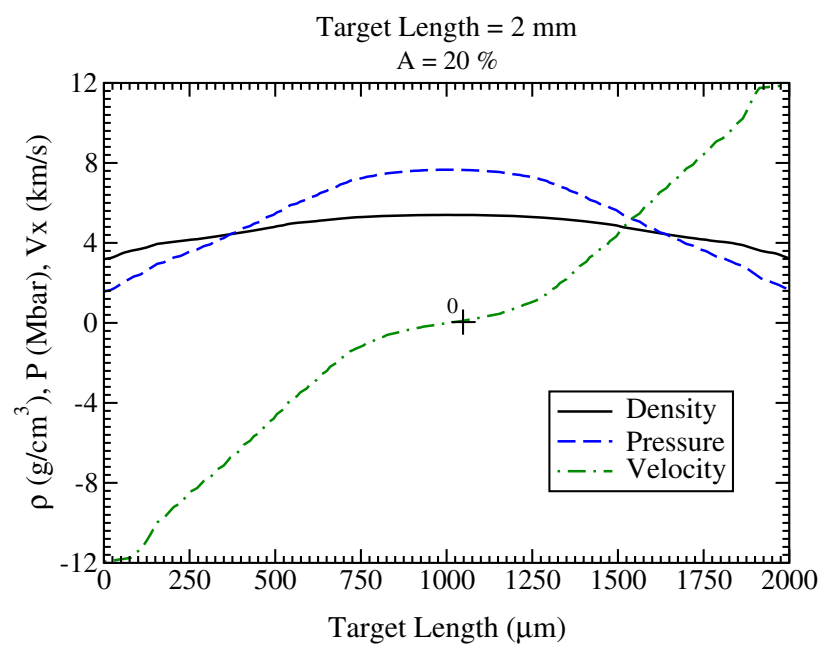




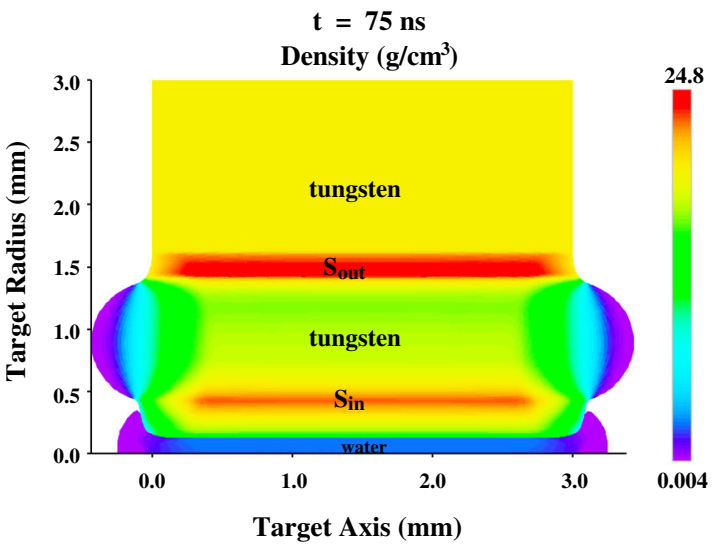

(a)

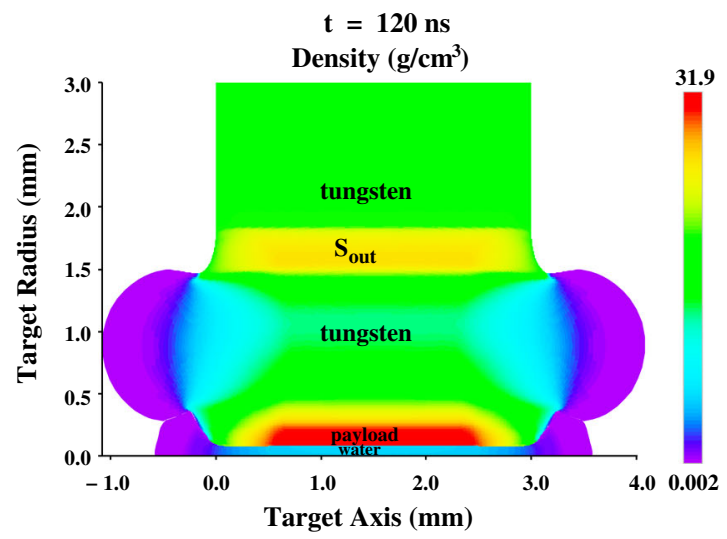

(c)

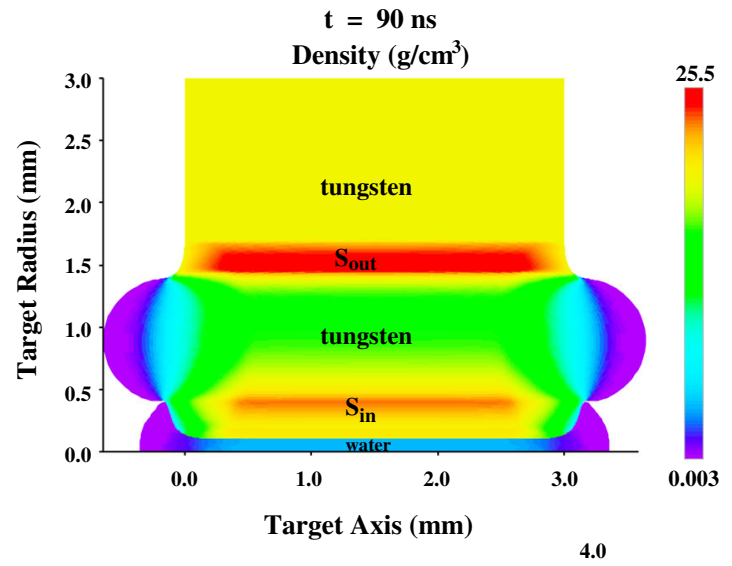

(b)

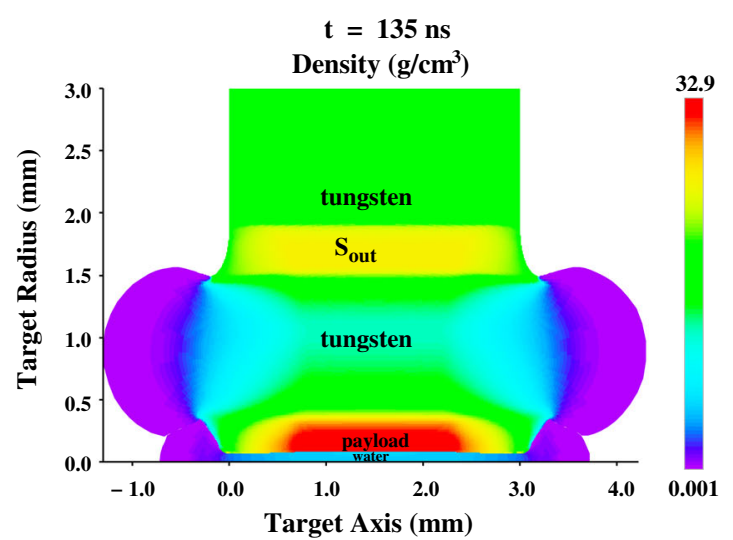

(d)

Fig. 8 Target density distribution at different times generated by BIG2 code, target length $=3 \mathrm{~mm}$, intensity $=5 \times 10^{11}$ uranium ions per bunch, particle energy $=1.5 \mathrm{GeV} / \mathrm{u}$, bunch length $=75 \mathrm{~ns}$, ring shaped focal spot, inner ring radius, $R_{1}=0.4 \mathrm{~mm}$, outer ring radius, $R_{2}=1.4 \mathrm{~mm}$, sample radius, $R_{i}=0.2 \mathrm{~mm}$, outer cylinder radius, $R_{O}=3 \mathrm{~mm}$ (see Fig. 2), foot amplitude, $A=0.2$ (see Fig. 3); $\mathbf{a}$ at $t=75 \mathrm{~ns}, \mathbf{b}$ at $t=90 \mathrm{~ns}$, $\mathbf{c}$ at $t=120 \mathrm{~ns}$ and $\mathbf{d}$ at $t=135 \mathrm{~ns}$

original target length. It is seen that the density and pressure are uniform, whereas the material velocity is zero within about $0.5 \mathrm{~mm}$ length around the center point of the axis. The density is about $5.4 \mathrm{~g} / \mathrm{cm}^{3}$ while the pressure is around $7.7 \mathrm{Mbar}$, which are close to the corresponding values obtained using 1D hydrodynamic model as noted in Table 2. This shows that using a 2-mm-long target, it may be possible to produce a small sample of warm dense water with steady state conditions.

\subsubsection{Target length $3 \mathrm{~mm}$}

The results obtained using a $3 \mathrm{~mm}$ long LAPLAS target are discussed in this sub-section. In Fig. 8, we present the simulated target density distribution at different times. Figure $8 \mathrm{a}$ is plotted at $t=75 \mathrm{~ns}$, which shows that the densities at the two shock fronts are the same as those in the previous two cases, whereas the fronts have bigger lengths due to a longer target. Same is true for Fig. 8b, which is plotted at $t=90 \mathrm{~ns}$.

Figure $8 \mathrm{c}$ is plotted at $t=120 \mathrm{~ns}$, which shows that the payload density is the same as in Fig. 6c, but the payload length is larger in the present case due to a longer target.

Figure $8 \mathrm{~d}$ is plotted at the time of maximum compression, $t=135 \mathrm{~ns}$. It is seen that the payload has been further compressed and has the same density as that in Fig. 6d. Moreover, the density and the pressure in the water sample are same as the corresponding 1D calculation results noted in Table 2. This means that the 1D approximation is valid in the inner region of the sample and the 2D effects are not important.

To have a quantitative understanding of the problem, we plot in Fig. 9 the density, the pressure and the material velocity along the inner $3 \mathrm{~mm}$ of the target axis at $t=135 \mathrm{~ns}$, the time of optimum compression. It is seen that the pressure and the density are very uniform, while the material velocity is zero in a $1.45 \mathrm{~mm}$ long zone that is symmetrically distributed around the center point 
Fig. 9 Density, pressure and material velocity along cylinder axis at $t=135 \mathrm{~ns}, L=3 \mathrm{~mm}$

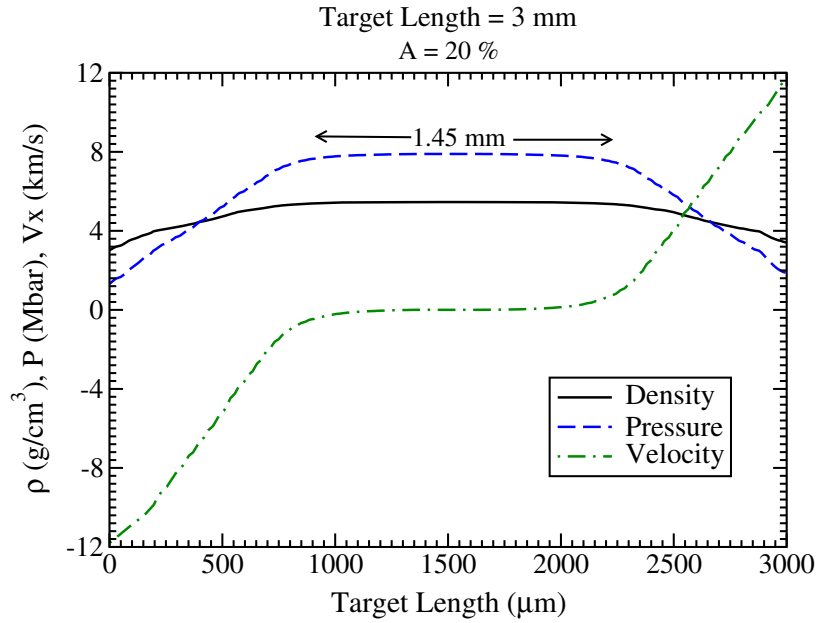

of the axis. The radius of this compressed zone is about $85 \mu \mathrm{m}$. This means that a reasonably large warm dense sample of water is generated using these beam and target parameters.

\subsubsection{Target length $4 \mathrm{~mm}$}

Finally, we discuss the results obtained considering a cylinder length of $4 \mathrm{~mm}$. In Fig. 10, the target density distributions generated by BIG2 at different times, are presented. Figure 10a shows that at $t=75 \mathrm{~ns}$, the densities at the two shock fronts are same as in the previous three cases, but the length of the fronts is larger due to the bigger target length. The same behavior is seen at $t=90 \mathrm{~ns}$ in Fig. 10b.

Figure 10c shows that at $t=120 \mathrm{~ns}$, the tamper payload density is the same as in Fig. 8c, which corresponds to $L=3 \mathrm{~mm}$, whereas the length of the payload is larger in the present case. This means that the length of the warm dense water sample produced in the LAPLAS implosion is larger as well.

Figure $10 \mathrm{~d}$ is plotted at $t=135 \mathrm{~ns}$, the time of maximum compression. The density in the compressed water region is $5.5 \mathrm{~g} / \mathrm{cm}^{3}$, while the pressure is $8 \mathrm{Mbar}$, which are the values achieved by 1D calculations presented in Table 2 .

In Fig. 11, we plot the density, pressure and the material velocity along the cylinder axis within the inner $4 \mathrm{~mm}$ zone, that is the original target length. It is seen that in the middle of the axis, there is a zone with uniform density and temperature, but with zero material velocity. The length of this zone is $2.55 \mathrm{~mm}$, which represents the longitudinal dimension of the warm dense water sample in steady state. The radius of this compressed water region is $85 \mu \mathrm{m}$. Such a configuration is ideal from the diagnostics point of view.

We note that in order to increase the resolution of the achieved density, pressure and material velocity at the time of maximum compression, we exclude the expanded part of the target and consider only the compressed region, which is equal to the original target length. This approach will be followed in similar figures in the rest of the paper.

\subsubsection{Comparison between results of different cases}

In Fig. 12a, we plot the density along the axis for cases with different target lengths, $L$, including $1,2,3$ and 4 mm, respectively, at $t=135 \mathrm{~ns}$, the time of maximum compression. It is seen that in case of $L=1 \mathrm{~mm}$, there is no region with uniform density, and the distribution is bell shaped with a maximum value of about $4 \mathrm{~g} / \mathrm{cm}^{3}$ at the top. This is because the cylinder aspect ratio is too small ( 2.5 for the water region), so the $2 \mathrm{D}$ affects are very important. Since the material is preheated, the lateral expansion occurs during the implosion that has adverse effect on the final compression.

The curve labeled with $L=2 \mathrm{~mm}$ shows a small flat region at the top which has a length of about $0.5 \mathrm{~mm}$. Moreover, the density in this region is same as given by the $1 \mathrm{D}$ simulation model, namely, $5.5 \mathrm{~g} / \mathrm{cm}^{3}$. This means that in case of a $2 \mathrm{~mm}$ long target, the rarefaction waves do not reach the target center over the time scale of the experiment.

The following curves labeled with $L=3$ and $4 \mathrm{~mm}$, show that the length of the flat region at the top increases with the target length. This study thus indicates that the LAPLAS target should be at least $3 \mathrm{~mm}$ long, although 4 mm length could be better.

In Fig. 12b, we plot the corresponding pressure curves, which show a similar behavior. We note that in order to see the characteristics of the density and pressure distributions in the longitudinal direction, in these figures we consider the entire axis that includes the expanded part of the target as well. This approach is used in similar figures in the following part of the paper.

It is also important to note that using other values of the constant power amplitude, $A$, namely, $0.1,0.3$ and 0.4 , respectively, the target response is very similar to that using $A=0.2$, as described above. Moreover, the physical parameters obtained in the warm 


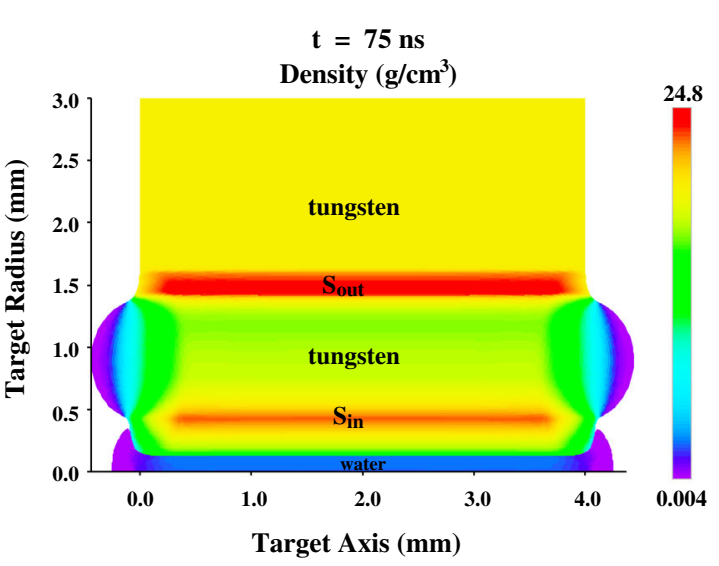

(a)

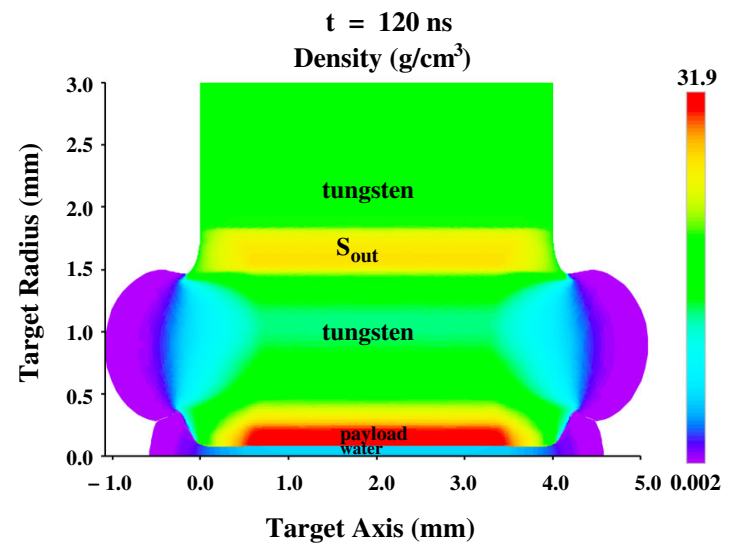

(c)

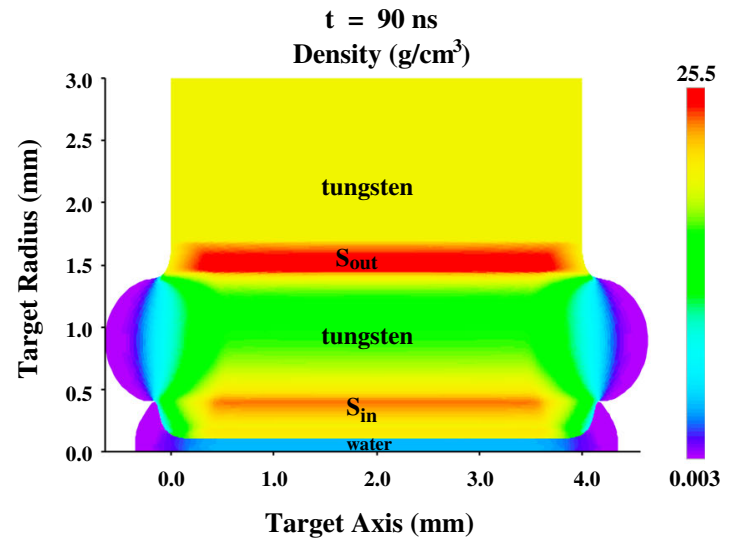

(b)

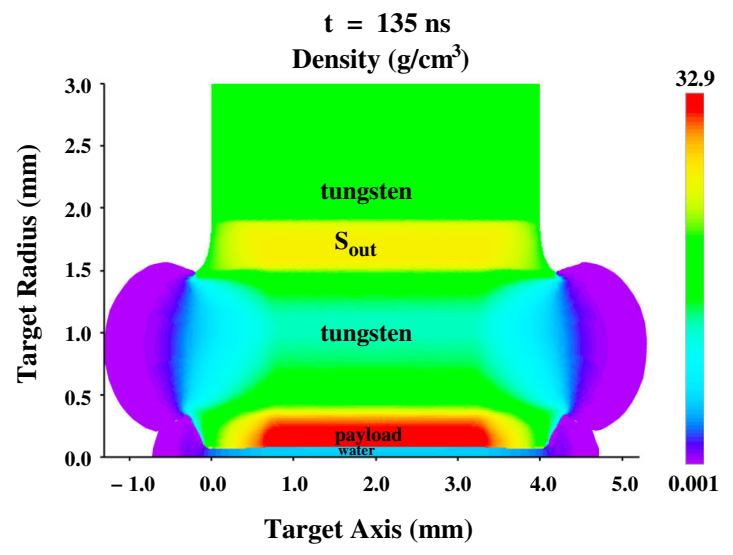

(d)

Fig. 10 Target density distribution at different times generated by BIG2 code, target length $=4 \mathrm{~mm}$, intensity $=5 \times 10^{11}$ uranium ions per bunch, particle energy $=1.5 \mathrm{GeV} / \mathrm{u}$, bunch length $=75 \mathrm{~ns}$, ring shaped focal spot, inner ring radius, $R_{1}=0.4 \mathrm{~mm}$, outer ring radius, $R_{2}=1.4 \mathrm{~mm}$, sample radius, $R_{i}=0.2 \mathrm{~mm}$, outer cylinder radius, $R_{O}=3 \mathrm{~mm}$ (see Fig. 2), foot amplitude, $A=0.2$ (see Fig. 3); $\mathbf{a}$ at $t=75 \mathrm{~ns}, \mathbf{b}$ at $t=90 \mathrm{~ns}$, $\mathbf{c}$ at $t=120 \mathrm{~ns}$ and $\mathbf{d}$ at $t=135 \mathrm{~ns}$

Fig. 11 Density, pressure and material velocity along cylinder axis, $L=4 \mathrm{~mm}$

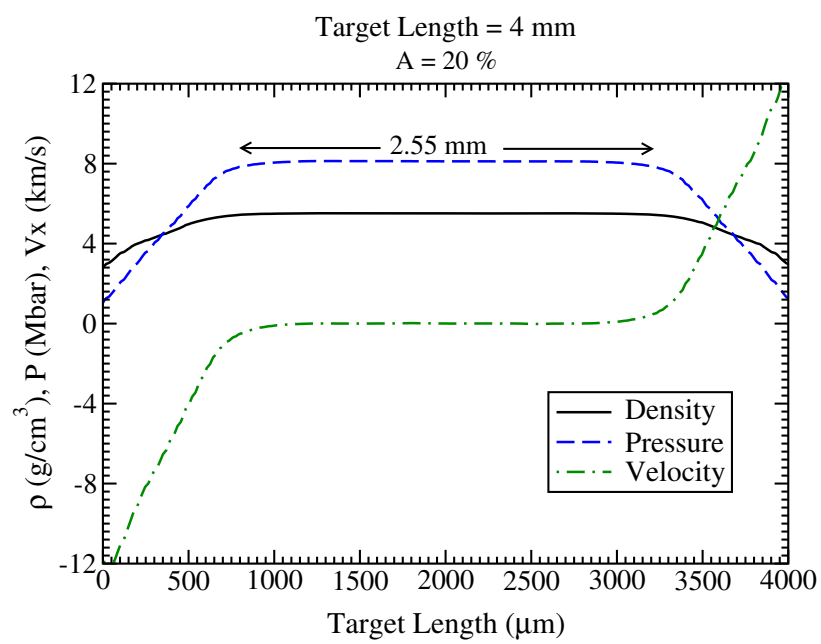




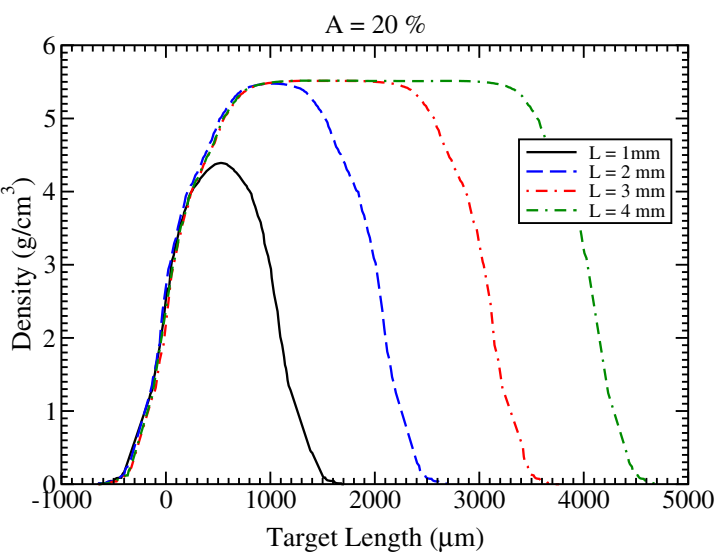

(a)

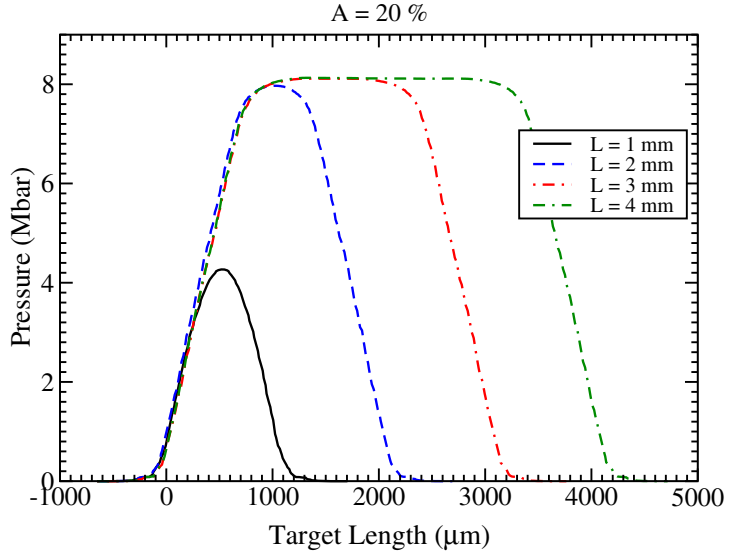

(b)

Fig. 12 Beam intensity $=5 \times 10^{11}$ uranium ions per bunch, particle energy $=1.5 \mathrm{GeV} / \mathrm{u}$, bunch length $=75 \mathrm{~ns}$, ring shaped focal spot, inner ring radius, $R_{1}=0.4 \mathrm{~mm}$, outer ring radius, $R_{2}=1.4 \mathrm{~mm}$, sample radius, $R_{i}=0.2 \mathrm{~mm}$, outer cylinder radius, $R_{O}=3 \mathrm{~mm}$ (see Fig. 2 ); a density versus axis at $t=135 \mathrm{~ns}$, b pressure versus axis at $t=135 \mathrm{~ns}$

dense water sample using $L=3$ and $4 \mathrm{~mm}$, are very close to their corresponding values noted in Table 2, that are obtained using 1D hydrodynamic version of the BIG2 code. The conclusions presented in this section are therefore valid over the entire parameter space considered in this study.

\subsection{Case 2}

Here we present simulation results considering Case 2 noted in Table 1, which means a sample radius, $R_{i}=0.2 \mathrm{~mm}$, an inner ring radius, $R_{1}=0.3 \mathrm{~mm}$ and an outer ring radius, $R_{2}=1.3 \mathrm{~mm}$. Again we consider the constant power foot, $A=0.2$, while four different values of target length, $L$ including $1,2,3$ and $4 \mathrm{~mm}$, respectively, are used. In the following, we present a summary of the results.

In Fig. 13, we plot the density, the pressure and the material velocity along the target axis (within inner $1 \mathrm{~mm}$ length) at $t=118 \mathrm{ns,}$ which is the time of maximum compression, for different values of $L$. Figure 13a shows that using $L=1 \mathrm{~mm}$, the density and the pressure are not uniform along the axis, while the material is expanding in the lateral direction with high velocity.

Figure 13b shows that using $L=2 \mathrm{~mm}$, the implosion leads to a $0.6 \mathrm{~mm}$ long zone, where the density and the pressure are uniform, while the material velocity is zero. The outer radius of this stationary zone is about $85 \mu \mathrm{m}$.

Figure 13c shows that using $L=3 \mathrm{~mm}$, leads to a longer steady state region of $1.6 \mathrm{~mm}$, whereas it is seen from Fig. 13d, that considering $L=4 \mathrm{~mm}$, this region extends to $2.6 \mathrm{~mm}$. The outer radius of the sample is again of the order of $85 \mu \mathrm{m}$.

In Fig. 14a, b, we present the density and the pressure along the entire target axis, respectively, at $t=118 \mathrm{~ns}$, for the four different values of the target length considered above. Figure 14a shows that in case of $L=1 \mathrm{~mm}$, the density is not uniform and has a bell shaped distribution with a maximum value of around $4.5 \mathrm{~g} / \mathrm{cm}^{3}$ at the top. A similar behavior is seen by the corresponding pressure curve in Fig. 14b.

It is further seen that using $L=2 \mathrm{~mm}$, a small region of uniform density and uniform pressure exists in the middle of the curves and the values of these parameters are close to those obtained using the 1D model (see Table 3 ). The following curves show that the size of the warm dense water sample increases with the target length. The corresponding pressure curves presented in Fig. 14b, show a similar behavior.

It is also to be noted that the overall behavior of the physical parameters obtained in calculations done using different values of the amplitude, A, is very similar. Also the values of these parameters for $L=3$ and $4 \mathrm{~mm}$ are identical to their corresponding 1D model values reported in Table 3. This study therefore again suggests that the length of the LAPLAS target for the considered beam and sample parameters should be from 3 to $4 \mathrm{~mm}$.

\subsection{Case 3}

Here we present simulation results considering Case 3 noted in Table 1, which means a sample radius, $R_{i}=0.3 \mathrm{~mm}$, an inner ring radius, $R_{1}=0.5 \mathrm{~mm}$ and an outer ring radius, $R_{2}=1.5 \mathrm{~mm}$. Again, we consider the constant power foot, $A=0.2$, while four different values of target length, $L$ including 1,2,3 and $4 \mathrm{~mm}$, respectively, are used. In the following, we present a summary of the results.

In Fig. 15, we plot the pressure, the density and the material velocity at $t=149$ ns (the time of maximum compression), using four different values of the target length, namely, $L=1,2,3$ and $4 \mathrm{~mm}$, respectively. We also assume that the amplitude, $A=0.2$ in all these cases. 


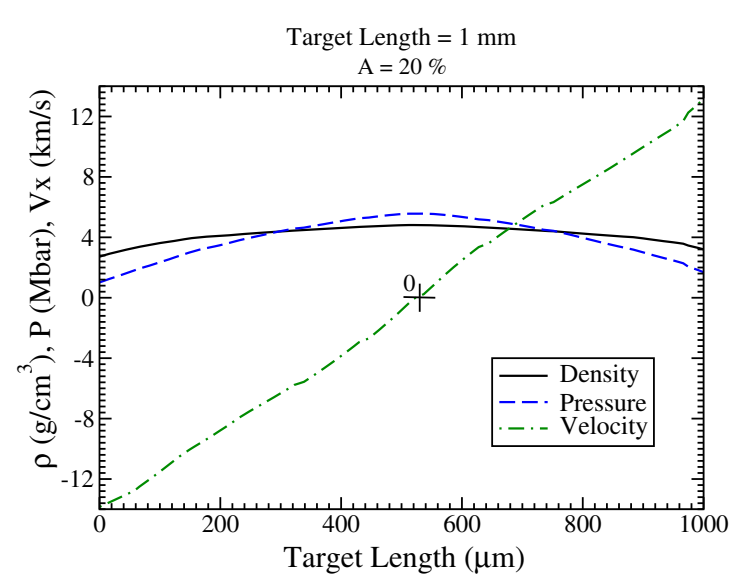

(a)

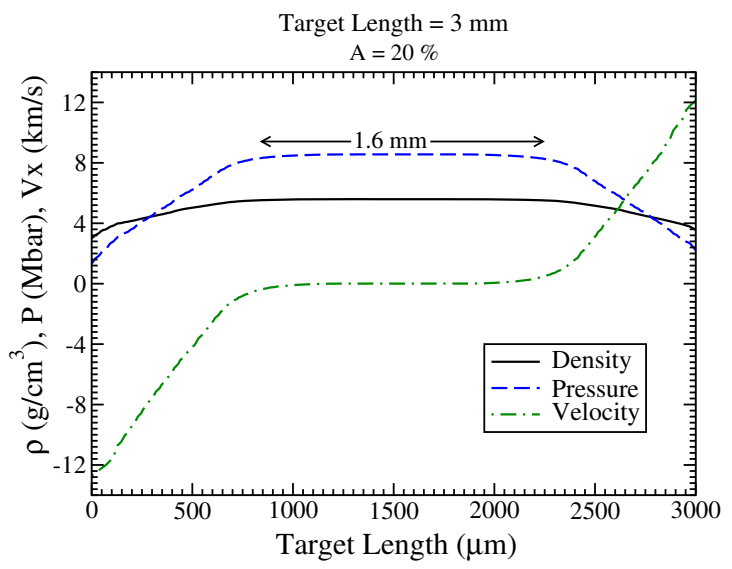

(c)

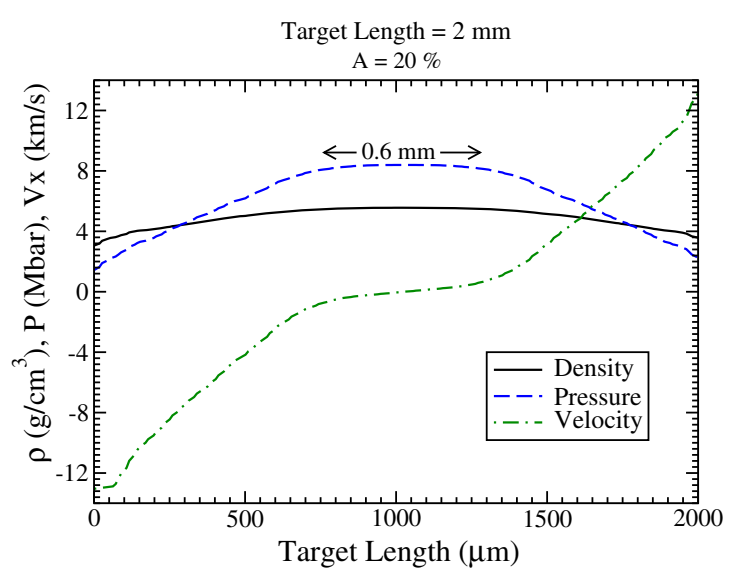

(b)

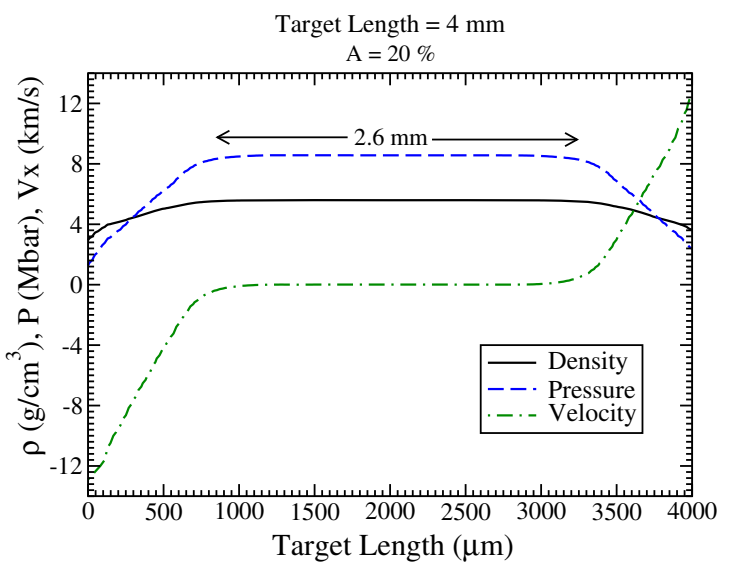

(d)

Fig. 13 Beam intensity $=5 \times 10^{11}$ uranium ions per bunch, particle energy $=1.5 \mathrm{GeV} / \mathrm{u}$, bunch length $=75 \mathrm{~ns}$, ring shaped focal spot, inner ring radius, $R_{1}=0.3 \mathrm{~mm}$, outer ring radius, $R_{2}=1.3 \mathrm{~mm}$, sample radius, $R_{i}=0.2 \mathrm{~mm}$, outer cylinder radius, $R_{O}=3 \mathrm{~mm}$ (see Fig. 2 ), foot amplitude, $A=0.2$ (see Fig. 3), at $t=118 \mathrm{~ns} ; \mathbf{a} L=1 \mathrm{~mm}, \mathbf{b ~ L}=2 \mathrm{~mm}, \mathbf{c} L=3 \mathrm{~mm}$ and $\mathbf{d} \mathrm{L}=4 \mathrm{~mm}$

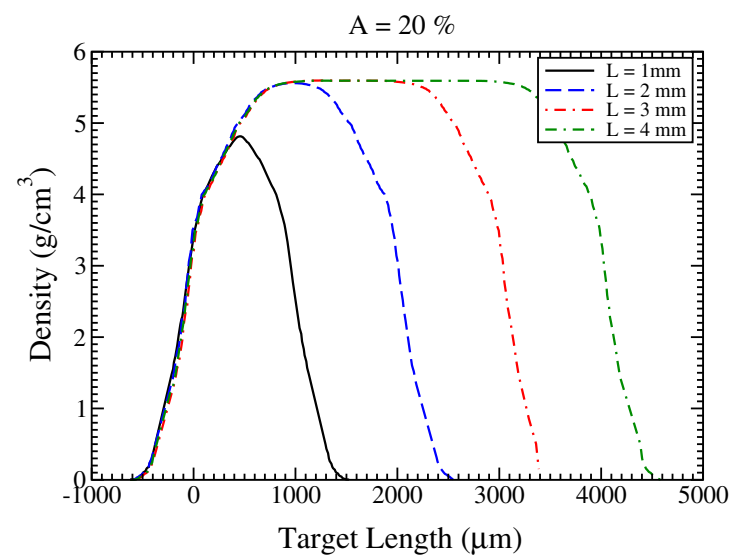

(a)

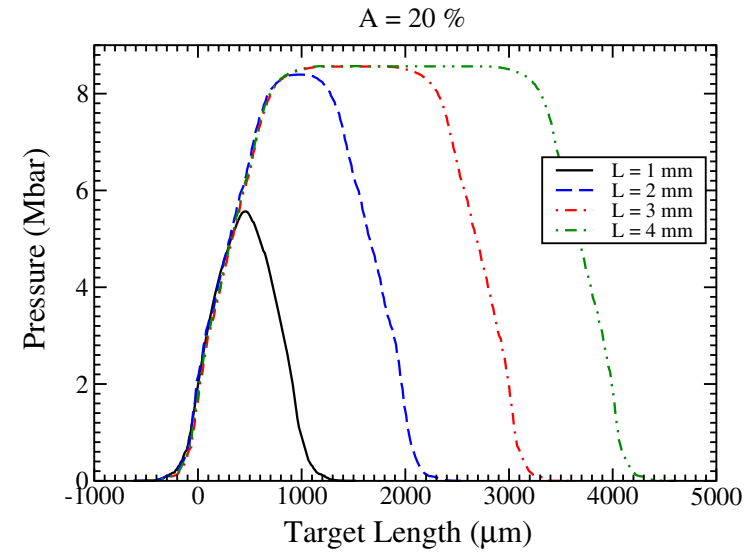

(b)

Fig. 14 Beam intensity $=5 \times 10^{11}$ uranium ions per bunch, particle energy $=1.5 \mathrm{GeV} / \mathrm{u}$, bunch length $=75 \mathrm{~ns}$, ring shaped focal spot, inner ring radius, $R_{1}=0.3 \mathrm{~mm}$, outer ring radius, $R_{2}=1.3 \mathrm{~mm}$, sample radius, $R_{i}=0.2 \mathrm{~mm}$, outer cylinder radius, $R_{O}=3 \mathrm{~mm}$ (see Fig. 2 ); a density versus axis at $t=118 \mathrm{~ns}$, b pressure versus axis at $t=118 \mathrm{~ns}$ 
Table 3 Optimum physical conditions in compressed water obtained using $1 \mathrm{D}$ hydrodynamic model, bunch intensity $=5 \times 10^{11}$ uranium ions, bunch length $=75 \mathrm{~ns}$, particle energy $=1.5 \mathrm{GeV} / \mathrm{u}$, sample radius, $R_{i}=0.2 \mathrm{~mm}$, annular focal spot with inner ring radius, $R_{1}=0.3 \mathrm{~mm}$, outer ring radius, $R_{2}=1.3 \mathrm{~mm}[64]$

\begin{tabular}{llllll}
\hline No & $A(\%)$ & $\rho\left(\mathrm{g} / \mathrm{cm}^{3}\right)$ & $P(\mathrm{Mbar})$ & $T(\mathrm{~K})$ & $t_{\text {impl }}(\mathrm{ns})$ \\
\hline 1 & 10 & 6.15 & 10.3 & $7 \times 10^{3}$ & 116 \\
2 & 20 & 5.62 & 8.6 & $10^{4}$ & $1.25 \times 10^{4}$ \\
3 & 30 & 5.3 & 7.6 & $1.5 \times 10^{4}$ \\
4 & 40 & 5.05 & 6.8 & 118 \\
\end{tabular}

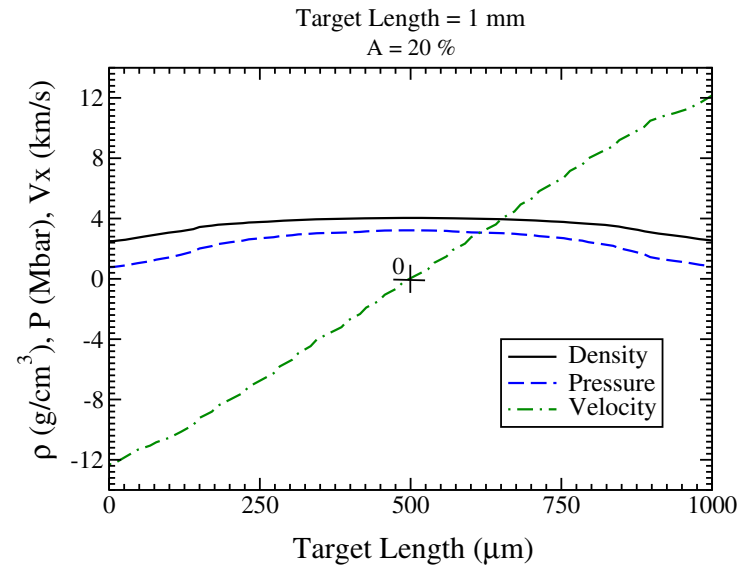

(a)

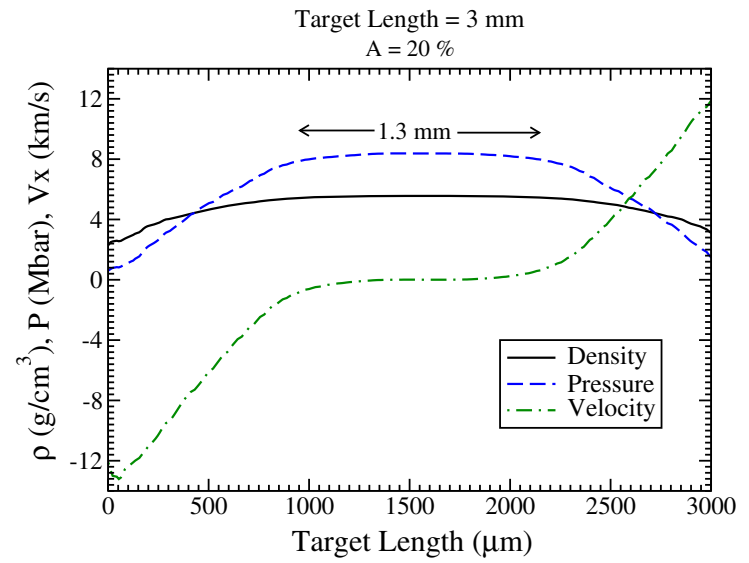

(c)

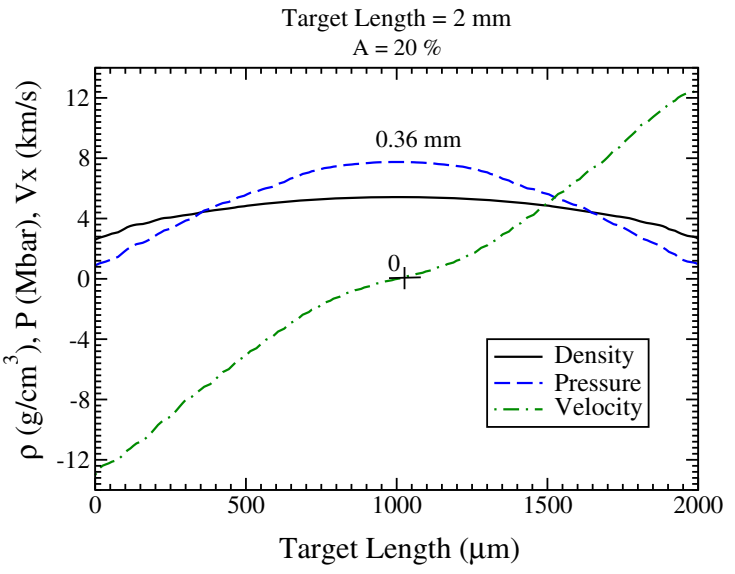

(b)

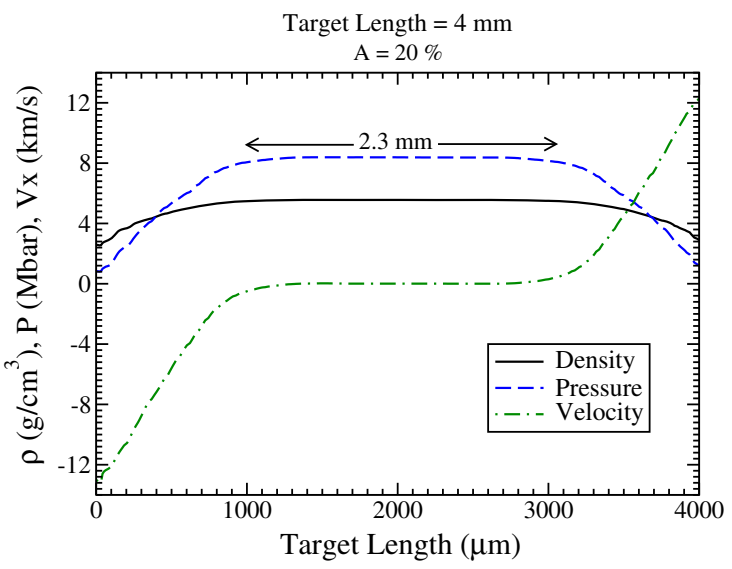

(d)

Fig. 15 Beam intensity $=5 \times 10^{11}$ uranium ions per bunch, particle energy $=1.5 \mathrm{GeV} / \mathrm{u}$, bunch length $=75 \mathrm{~ns}$, ring shaped focal spot, inner ring radius, $R_{1}=0.5 \mathrm{~mm}$, outer ring radius, $R_{2}=1.5 \mathrm{~mm}$, sample radius, $R_{i}=0.3 \mathrm{~mm}$, outer cylinder radius, $R_{O}=3 \mathrm{~mm}$ (see Fig. 2), foot amplitude, $A=0.2$ (see Fig. 3), at $t=149 \mathrm{~ns} ; \mathbf{a} L=1 \mathrm{~mm} \mathrm{~b} L=2 \mathrm{~mm}, \mathbf{c} L=3 \mathrm{~mm}$ and $\mathbf{d} L=4 \mathrm{~mm}$

Figure 15a shows that considering $1 \mathrm{~mm}$ long target, the material velocity is zero at the middle of the axis, while it increases rapidly on both sides. This shows that sample material is expanding fast and there is no steady state region. Moreover, the density and the pressure have much lower values as compared to their corresponding values predicted by $1 \mathrm{D}$ hydrodynamic model (see Table 4). It is therefore concluded that a $1 \mathrm{~mm}$ length of the target is not suitable for this experiment.

Figure $15 \mathrm{~b}$ represents the case $L=2 \mathrm{~mm}$. It is seen that a small stationary zone $(0.36 \mathrm{~mm}$ long $)$ at the middle of the axis exists, where the density and the pressure are uniform with values close to the corresponding $1 \mathrm{D}$ calculations. This means that the rarefaction waves entering from the two faces of the cylinder have still not arrived at the center by this time.

Figure 15c shows that in case of $L=3 \mathrm{~mm}$, the length of the compressed warm dense water steady state zone is $1.3 \mathrm{~mm}$, which is sufficient for the experimental studies. The length of this zone becomes $2.3 \mathrm{~mm}$ using $L=4 \mathrm{~mm}$, as shown in Fig. 15d.

The outer radius of the steady state compressed sample is around $125 \mu \mathrm{m}$. 
Table 4 Optimum physical conditions in compressed water obtained using 1D hydrodynamic model, bunch intensity $=5 \times 10^{11}$ uranium ions, bunch length $=75 \mathrm{~ns}$, particle energy $=1.5 \mathrm{GeV} / \mathrm{u}$, sample radius, $R_{i}=0.3 \mathrm{~mm}$, annular focal spot with inner ring radius, $R_{1}=0.5 \mathrm{~mm}$, outer ring radius, $R_{2}=1.5 \mathrm{~mm}[64]$

\begin{tabular}{llllll}
\hline No & $A(\%)$ & $\rho\left(\mathrm{g} / \mathrm{cm}^{3}\right)$ & $P($ Mbar $)$ & $T(\mathrm{~K})$ & $t_{\text {impl }}(\mathrm{ns})$ \\
\hline 1 & 10 & 6.08 & 9.82 & $6.3 \times 10^{3}$ \\
2 & 20 & 5.55 & 8.2 & $9 \times 10^{3}$ \\
3 & 30 & 5.2 & 7.1 & $1.15 \times 10^{4}$ \\
4 & 40 & 4.9 & 6.12 & $1.35 \times 10^{4}$ & 149 \\
\hline
\end{tabular}

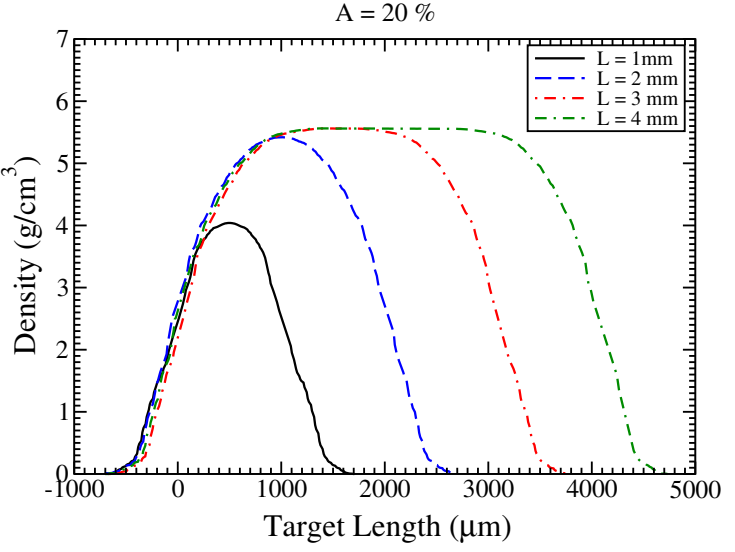

(a)

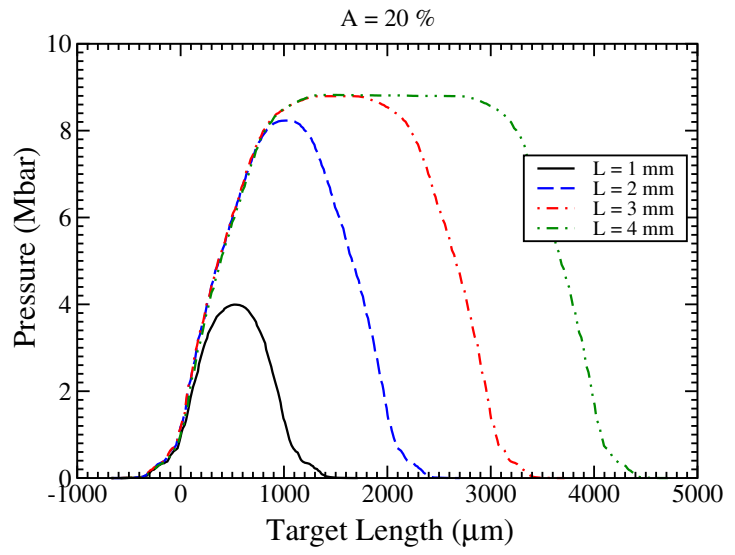

(b)

Fig. 16 Beam intensity $=5 \times 10^{11}$ uranium ions per bunch, particle energy $=1.5 \mathrm{GeV} / \mathrm{u}$, bunch length $=75 \mathrm{~ns}$, ring shaped focal spot, inner ring radius, $R_{1}=0.5 \mathrm{~mm}$, outer ring radius, $R_{2}=1.5 \mathrm{~mm}$, sample radius, $R_{i}=0.3 \mathrm{~mm}$, outer cylinder radius, $R_{O}=3 \mathrm{~mm}$ (see Fig. 2 ); a density versus axis at $t=149 \mathrm{~ns}$, b pressure versus axis at $t=149 \mathrm{~ns}$

In Fig. 16a, we plot the target density along the axis at $t=149 \mathrm{~ns}$, considering $A=0.2$ and using $L=1,2,3$ and $4 \mathrm{~mm}$, respectively. The corresponding pressure curves are presented in Fig. 16b. It is seen in Fig. 16a that using $L=1 \mathrm{~mm}$, the density curve is bell shaped and there is no uniform density region. The maximum density at the top of the curve is around $4 \mathrm{~g} / \mathrm{cm}^{3}$, which is lower than the corresponding value predicted by 1D model presented in Table 4. Similar behavior is shown by the corresponding pressure curve in Fig. 16b.

The density and the pressure curves for the case $L=2 \mathrm{~mm}$ are almost bell shaped with small flat regions at the top. It is also seen that the maximum parameter values are lower than the corresponding $1 \mathrm{D}$ model values. It is further seen that for the cases using target length of 3 and $4 \mathrm{~mm}$, the dimensions of the flat part of the curves increase, whereas the parameter values become equal to those achieved by 1D model noted in Table 4.

It is also to be noted that the simulation results obtained using other values of A show a similar behavior. One can therefore conclude, that the length of the LAPLAS target for the beam and sample parameters considered in this study should also be 3 to $4 \mathrm{~mm}$.

\subsection{Case 4}

Here we present simulation results considering Case 4 noted in Table 1 , which means a sample radius, $R_{i}=0.3 \mathrm{~mm}$, an inner ring radius, $R_{1}=0.4 \mathrm{~mm}$ and an outer ring radius, $R_{2}=1.4 \mathrm{~mm}$. Again we consider the constant power foot, $A=0.2$, while four different values of target length, $L$ including 1,2, 3 and $4 \mathrm{~mm}$, respectively, are used. In the following, we present a summary of the results.

In Fig. 17, we present the pressure, the density and the material velocity at $t=137 \mathrm{~ns}$ (the time of maximum compression), using four different values of the target length, namely, $L=1,2,3$ and $4 \mathrm{~mm}$, respectively. We also assume that the amplitude, $A=0.2$ in all these cases.

Figure 17a represents the case, $L=1 \mathrm{~mm}$. It is seen that the density and the pressure are not uniform along the axis and have lower values compared to the corresponding 1D model values reported in Table 5. Moreover, the material velocity is zero only at the center of the axis, while it increases on either side of this point, which indicates that the entire sample material is rapidly expanding.

Figure $17 \mathrm{~b}$ corresponds to the case, $L=2 \mathrm{~mm}$, which shows that the density and the pressure are uniform in a small central zone on the axis. However, the material velocity is non-zero, except at the central point. This means that the entire sample material is expanding. 


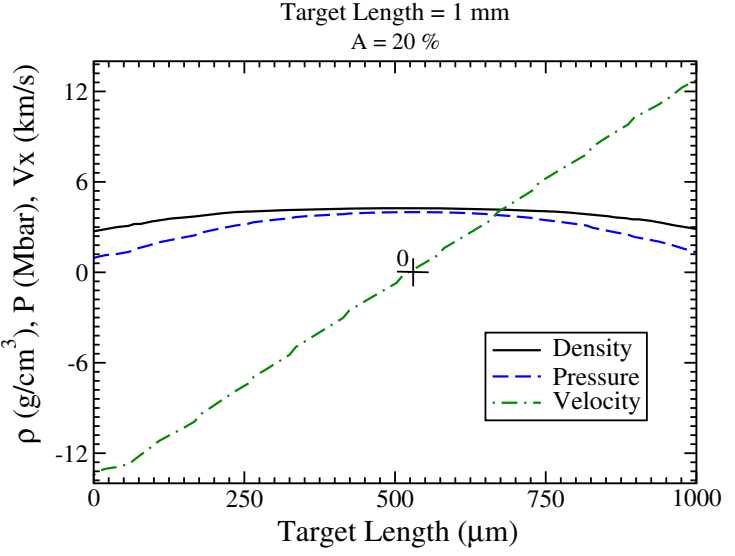

(a)

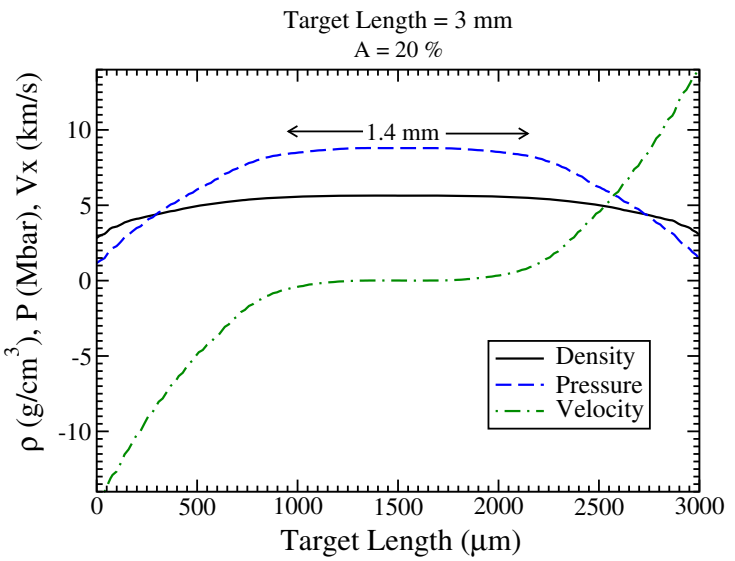

(c)

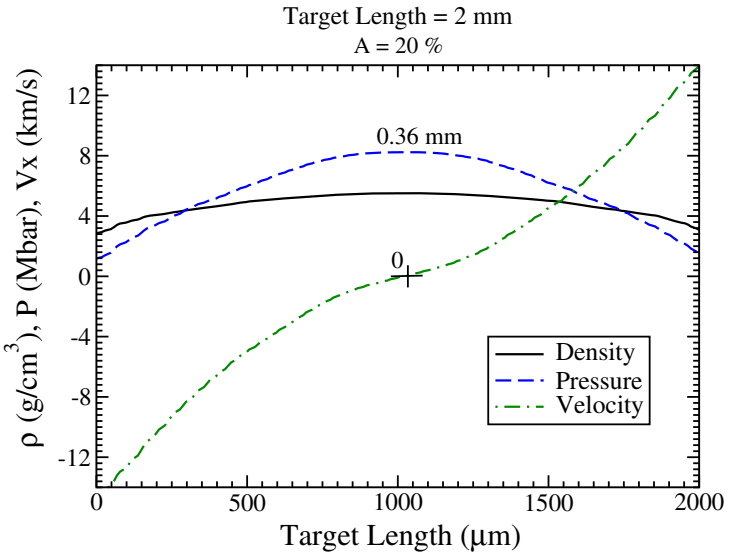

(b)

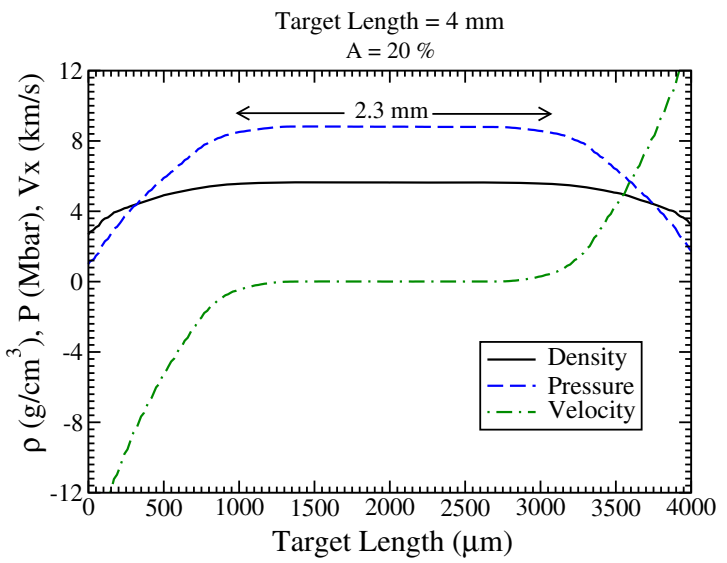

(d)

Fig. 17 Beam intensity $=5 \times 10^{11}$ uranium ions per bunch, particle energy $=1.5 \mathrm{GeV} / \mathrm{u}$, bunch length $=75 \mathrm{~ns}$, ring shaped focal spot, inner ring radius, $R_{1}=0.4 \mathrm{~mm}$, outer ring radius, $R_{2}=1.4 \mathrm{~mm}$, sample radius, $R_{i}=0.3 \mathrm{~mm}$, outer cylinder radius, $R_{O}=3 \mathrm{~mm}$ (see Fig. 2), foot amplitude, $A=0.2$ (see Fig. 3), at $t=137 \mathrm{~ns} ; \mathbf{a} L=1 \mathrm{~mm}, \mathbf{b} L=2 \mathrm{~mm}, \mathbf{c} L=3 \mathrm{~mm}$ and $\mathbf{d} \mathrm{L}=4 \mathrm{~mm}$

Table 5 Optimum physical conditions in compressed water obtained using 1D hydrodynamic model, bunch intensity $=5 \times 10^{11}$ uranium ions, bunch length $=75 \mathrm{~ns}$, particle energy $=1.5 \mathrm{GeV} / \mathrm{u}$, sample radius, $R_{i}=0.3 \mathrm{~mm}$, annular focal spot with inner ring radius, $R_{1}=0.4 \mathrm{~mm}$, outer ring radius, $R_{2}=1.4 \mathrm{~mm}[64]$

\begin{tabular}{llllll}
\hline No & $A(\%)$ & $\rho\left(\mathrm{g} / \mathrm{cm}^{3}\right)$ & $P(\mathrm{Mbar})$ & $T(\mathrm{~K})$ & $t_{\text {impl }}(\mathrm{ns})$ \\
\hline 1 & 10 & 6.1 & 10.4 & $6.9 \times 10^{3}$ & 136 \\
2 & 20 & 5.7 & 8.75 & $10^{4}$ & $1.26 \times 10^{4}$ \\
3 & 30 & 5.3 & 7.6 & $1.5 \times 10^{4}$ & 138 \\
4 & 40 & 4.96 & 6.5 & 139 \\
\hline
\end{tabular}

Figure $17 \mathrm{c}$ represents the case, $L=3 \mathrm{~mm}$. It is seen that the density and the pressure are uniform within $1.4 \mathrm{~mm}$ at the center of the target. The material velocity in this region is zero and the radius of this stationary zone is about $125 \mu \mathrm{m}$.

Figure $17 \mathrm{~d}$ corresponds to the case, $L=4 \mathrm{~mm}$, which shows that the length of the steady state zone is now $2.3 \mathrm{~mm}$. The radius of this zone is also $125 \mu \mathrm{m}$. It is also to be noted that the density and the pressure values in the steady zones in Fig. 17c, d, respectively, are same as predicted by $1 \mathrm{D}$ calculations (see Table 5).

In Fig. 18a, we present the density along the entire axis at $t=137 \mathrm{~ns}$, which is the time of optimum compression, considering different values of the target length, including, $1,2,3$ and $4 \mathrm{~mm}$, respectively. Also, the amplitude, A is assumed to be 0.2 . The corresponding pressure curves are shown in Fig. 18b.

The respective density and the pressure curves presented in Fig. 18a, b, show the same behavior as the corresponding curves in Fig. 16a, b, respectively. It is also to be noted that different values of the amplitude, A, lead to a similar target response related 


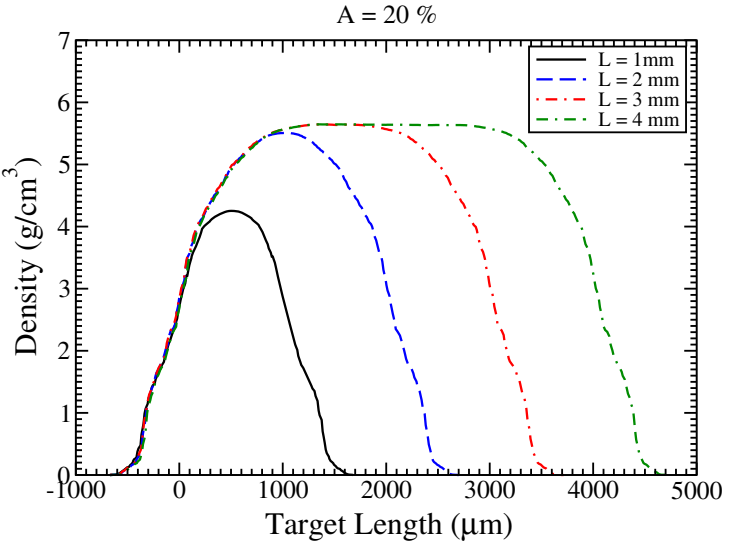

(a)

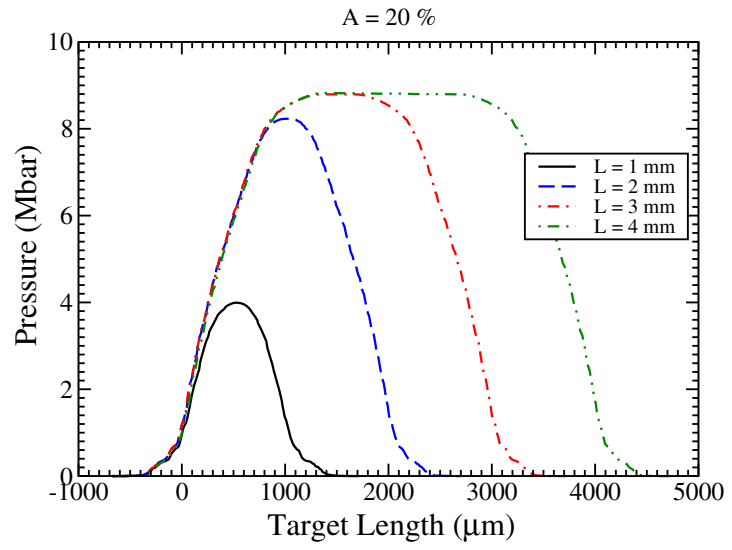

(b)

Fig. 18 Beam intensity $=5 \times 10^{11}$ uranium ions per bunch, particle energy $=1.5 \mathrm{GeV} / \mathrm{u}$, bunch length $=75 \mathrm{~ns}$, ring shaped focal spot, inner ring radius, $R_{1}=0.4 \mathrm{~mm}$, outer ring radius, $R_{2}=1.5 \mathrm{~mm}$, sample radius, $R_{i}=0.3 \mathrm{~mm}$, outer cylinder radius, $R_{o}=3 \mathrm{~mm}$ (see Fig. 2 ); a density versus axis at $t=137 \mathrm{~ns}$, b pressure versus axis at $t=137 \mathrm{~ns}$

to the finally achieved physical parameters. It is thus concluded that optimum length of the target using the above parameters is recommended to be 3 to $4 \mathrm{~mm}$.

\section{Conclusions}

This paper shows with the help of 2D hydrodynamic simulations that intense ion beams can be employed to do planetary physics research in the laboratory. An experimental scheme, named LAPLAS, has been proposed as part of the HED physics program to be carried out at the FAIR international facility at Darmstadt, Germany. The flexibility of this scheme is underlined by the fact that in these experiments, different sample materials including, hydrogen, water, carbon and iron can be used to generate the core conditions of hydrogen-rich, water-rich, carbon-rich and iron-rich planets, respectively. In the present study, we carried out detailed 2D hydrodynamic simulations of compression of water with emphasis on determining an optimum target length, which is important for the facilitation of the diagnostics. A wide range of the beam and target parameters has been considered that shows that the results are insensitive to large variations in these parameters, which implies that the scheme is robust and stable. In this paper we present results obtained using a uranium beam with a particle energy of $1.5 \mathrm{GeV} / \mathrm{u}$, whereas the bunch intensity is $5 \times 10^{11}$ ions and the bunch length is $75 \mathrm{~ns}$. Different values of the sample and the payload mass, respectively, have been considered. Moreover, different levels of the sample preheat are simulated using different values of the parameter A (see Fig. 3). These studies have shown that over the considered range of the input parameters, the target length should be 3 to $4 \mathrm{~mm}$ in order to avoid deleterious influence of the $2 \mathrm{D}$ effects on the sample implosion. An initial target length in this range provides a warm dense water sample having a length of 1.5 to $2.5 \mathrm{~mm}$, and a radius of 85 to $125 \mu \mathrm{m}$, with fairly uniform physical conditions.

Funding Open Access funding enabled and organized by Projekt DEAL.

Data Availability Statement The data that support the finding of this study are available within the article.

Open Access This article is licensed under a Creative Commons Attribution 4.0 International License, which permits use, sharing, adaptation, distribution and reproduction in any medium or format, as long as you give appropriate credit to the original author(s) and the source, provide a link to the Creative Commons licence, and indicate if changes were made. The images or other third party material in this article are included in the article's Creative Commons licence, unless indicated otherwise in a credit line to the material. If material is not included in the article's Creative Commons licence and your intended use is not permitted by statutory regulation or exceeds the permitted use, you will need to obtain permission directly from the copyright holder. To view a copy of this licence, visit http://creativecommons.org/licenses/by/4.0/.

\section{References}

1. D. Valencia, R.J. O'Connel, D. Sasselov, Icar 181, 545 (2006)

2. D. Valencia, D. Sasselov, R.J. O’Connel, ApJ 665, 1413 (2007)

3. D. Valencia, D. Sasselov, R.J. O'Connel, ApJ 656, 545 (2007)

4. C. Sotin, O. Grasset, A. Mocquet, Icar 191, 337 (2007)

5. D.C. Swift, J.G. Eggert, D.G. Hicks, S. Hamel, K. Caspersen, E. Schwegler, G.W. Collins, N. Nettelmann, G.J. Ackland, ApJ 744, 59 (2012)

6. H.-S. Park et al., Phys. Plasmas 15, 072705 (2008) 
7. T. Duffy, N. Madhusudhan, K.K.M. Lee, In: Treatise on Geophysics, 2nd edn, G. Schubert (Oxford Elsevier) 149 (2015)

8. M.J. Kuchner, ApJ 596, L105 (2003)

9. A. Leger, F. Selsis, C. Sotin, T. Guillot, D. Despois, D. Mawet, M. Ollivier, A. Labeque, C. Valette, F. Brachet et al., Icar 169, 499 (2004)

10. D. Ehrenreich, A.L. des Etangs, J.-P. Beaulieu, O. Grasset, ApJ 651, 535 (2006)

11. L. Zeng, D. Sasselov, ApJ 784: 96 (2914)

12. N. Nettelmann, U. Kramm, R. Redmer, R. Neuhaeuser, A \& A 523, A26 (2010)

13. N. Nettelmann, K. Wang, J.J. Fortney, S. Hamel, S. Yellamilli, M. Bethkenhagen, R. Redmer, Icar 275, 107 (2016)

14. M. Podolak, R. Helled, G. Schubert, MNRAS 487, 2653 (2019)

15. L. Scheibe, N. Nettelmann, R. Redmer, A \& A 632, A70 (2019)

16. T. Guillot, D. Gautier, In: Treatise on Geophysics, 2nd edn., ed. G. Schubert (Oxford Elsevier) 529 (2015)

17. A. Ng, Int. J. Q. Chem. 112, 150 (2012)

18. P.D. Drake, Phys. Plasmas 16, 055501 (2009)

19. V.E. Fortov, Extreme States of Matter (Springer, Heidelberg, 2016)

20. H.K. Mao, R.J. Hemley, Rev. Mod. Phys. 66, 671 (1994)

21. C. Narayan, H. Luo, A.L. Ruoff, Nature 393, 46 (1998)

22. R. Boehler, Rev. Geophys. 38, 221 (2000)

23. A.V. Altschuler, R.F. Trunen, K.K. Krupnikov, N.V. Panov., Physics-Uspekhi, 39, 539 (1996)

24. M. van Thiel, Compendium of Shock Wave Data, UCRL-50108 (1977)

25. V.E. Fortov, V.Y. Ternovoi, M.V. Zernokletov, M.A. Mochalov, A.L. Michailov, A.S. Filimonov, A.A. Pyalling, V.B. Mintsev, V.K. Gryaznov, I.L. Iosilevski, JETP 97, 259 (2003)

26. M.D. Knudson, D.L. Hanson, J.E. Bailey, C.A. Hall, J.R. Asay, W.W. Anderson, Phys. Rev. Lett. 87, 225501 (2001)

27. Th. Loewer, R. Sigel, K. Eidmann, B. Foeldes, S. Hueller, J. Massen, G.D. Tsakiris, S. Witkowski, W. Preuss, H. Nishimura et al., Phys. Rev. Lett. 72, $3186(1994)$

28. R. Cauble, D.W. Fillion, T.J. Hoover, N.C. Holmes, J.D. Kilkenny, R.W. Lee, Phys. Rev. Lett. 70, 2102 (1993)

29. M. Koenig, B. Faral, J.M. Boudenne, D. Batani, A. Benuzzi, S. Bossi, C. Remond, J.P. Perrine, M. Temporal, S. Atzeni, Phys. Rev. Lett. 74, 2260 (1995)

30. A.S. Vladimirov, N.P. Voloshin, V.N. Nogin, A.V. Petrovtsev, V.A. Simoneko, JETP 39, 82 (1984)

31. W.F. Henning, Nucl. Inst. Methods B 214, 2011 (2004)

32. P.J. Spiller, R. Balss, A. Bleile, L.H.J. Bozyk, J. Ceballos Velasco, T. Eisel, E.S. Fisher, P. Fork, P. Huelsmann, M. Kauschke et al., 'Status of the FAIR Synchrotron Projects SIS18 upgrade and SIS100'. in Proc. of IPAC2014, Dresden, Germany (2014)

33. J. Eshke, J. Phys. G 31, 967 (2005)

34. M. Durante, P. Indelicato, B. Johnson, V. Koch, K. Langanke, U.G. Miessner, E. Nappi, T. Nilsson, Th. Stoehlker, E. Widmann et al., Phys. Scr. 94, 033001 (2019)

35. N.A. Tahir, K.A. Long, Laser Part. Beams 2, 371 (1984)

36. N.A. Tahir, A. Kozyreva, P. Spiller, P. Spiller, D.H.H. Hoffmann, A. Shutov, Phys. Rev. E 63, 036407 (2001)

37. N.A. Tahir, P. Spiller, A. Shutov, I.V. Lomonosov, V. Grayaznov, A.R. Piriz, G. Wouchuk, C. Deutsch, V.E. Fortov, D.H.H. Hoffmann et al., Nucl. Inst. Methods A 577, 238 (2007)

38. N.A. Tahir, P. Spiller, S. Udrea, O.D. Cortazar, C. Deutsch, V.E. Fortov, V. Gryazanov, D.H.H. Hoffmann, I.V. Lomonosov, P. Ni et al., Nucl. Inst. Methods B 245, 85 (2006)

39. N.A. Tahir, A. Shutov, D. Varentsov, P. Spiller, S. Udrea, D.H.H. Hoffmann, I.V. Lomonosov, J. Wieser, M. Kirk, A.R. Piriz et al., Phys. Rev. ST Accel. Beams 6, 020101 (2003)

40. N.A. Tahir, B. Goddard, V. Kain, R. Schmidt, A. Shutov, I.V. Lomonosov, A.R. Piriz, M. Temporal, D.H.H. Hoffmann, V.E. Fortov, J. Appl. Phys. 97, $083532(2005)$

41. N.A. Tahir, Th. Stoehlker, A. Shutov, I.V. Lomonosov, V.E. Fortov, M. French, N. Nettelmann, R. Redmer, A.R. Piriz, C. Deutsch et al., New J. Phys. 12, $073022(2010)$

42. N.A. Tahir, A. Shutov, A.P. Zharkov, A.R. Piriz, Th. Stoehlker, Phys. Plasmas 18, 032704 (2011)

43. N.A. Tahir, P. Neumayer, A. Shutov, A.R. Piriz, I.V. Lomonosov, V. Bagnoud, S.A. Piriz, C. Deutsch, Contribu. Plasma Phys. 59, e201800143 (2019)

44. U.N. Funk, R. Bock, M. Dornik, M. Geissel, M. Stetter, S. Stoewe, N.A. Tahir, D.H.H. Hoffmann, Nucl. Inst. Methods A 415, 68 (1998)

45. D.H.H. Hoffmann, R. Bock, A.Y. Faenov, U. Funk, M. Geissel, U. Neuner, T.A. Pikuz, F. Rosmej, M. Roth, W. Suess, N.A. Tahir, A. Tauschwitz, Nucl. Inst. Methods B 161, 9 (2000)

46. S.A. Piriz, A.R. Piriz, N.A. Tahir, Phys. Rev. E 95, 053108 (2017)

47. S.A. Piriz, A.R. Piriz, N.A. Tahir, Phys. Fluids 30, 111703 (2018)

48. S.A. Piriz, A.R. Piriz, N.A. Tahir, J. Fluid Mech. 867, 1012 (2019)

49. S.A. Piriz, A.R. Piriz, N.A. Tahir, Phys. Rev. E 97, 043106 (2018)

50. J.J. Lopez Cela, A.R. Piriz, M.C. Serna Moreno, N.A. Tahir, Laser Part. Beams 24, 427 (2006)

51. J.J. Barnard, J. Armijo, R.M. More, A. Frieman, I. Kaganovich, B.G. Logan, M.M. Marinak, G.E. Penn, A.B. Sefkow, P. Santhanam et al., Nucl. Inst. Methods A 577 (2007)

52. N.A. Tahir, I.V. Lomonosov, B. Borm, A.R. Piriz, A. Shutov, P. Neumayer, V. Bagnoud, S.A. Piriz, ApJS 232, 1 (2017)

53. N.A. Tahir, A. Shutov, I.V. Lomonosov, A.R. Piriz, P. Neumayer, V. Bagnoud, S.A. Piriz, ApJS 238, 27 (2018)

54. N.A. Tahir, I.V. Lomonosov, B. Borm, A.R. Piriz, P. Neumayer, A. Shutov, V. Bagnoud, S.A. Piriz, Contrib. Plasma Phys. 57, 493 (2017)

55. N.A. Tahir, A. Shutov, A.R. Piriz, P. Neumayer, I.V. Lomonosov, V. Bagnoud, S.A. Piriz, Contrib. Plasma Phys. 59, e201800135 (2019)

56. N.A. Tahir, C. Deutsch, V.E. Fortov, V. Gryazanov, D.H.H. Hoffmann, M. Kulish, I.V. Lomonosov, V. Mintsev, P. Ni, D. Nikolaev et al., Phys. Rev. Lett. 95, $035001(2005)$

57. N.A. Tahir, A. Shutov, I.V. Lomonosov, A.R. Piriz, G. Wouchuk, C. Deutsch, D.H.H. Hoffmann, V.E. Fortov, High Energy Density Phys. 2, 21 (2006)

58. N.A. Tahir, P. Neumayer, I.V. Lomonosov, A. Shutov, V. Bagnoud, A.R. Piriz, S.A. Piriz, C. Deutsch, Phys. Rev. E 101, 023202 (2020)

59. K. Schoenberg, V. Bagnoud, A. Blazevic, V.E. Fortov, D.O. Gerike, A. Golubev, D.H.H. Hoffmann, D. Kraus, I.V. Lomonosov, V. Mintsev et al., Phys. Plasmas 27, 043103 (2020)

60. A.R. Piriz, M. Temporal, J.J. Lopez Cela, N.A. Tahir, D.H.H. Hoffmann, Plasma Phys. Controll. Fusion 45, 1733 (2003)

61. S. Minaev, N. Alexeev, A. Golubev, D.H.H. Hoffmann, T. Kulevoy, B. Sharkov, A. Sitnikov, N.A. Tahir, D. Varentsov, Nucl. Instr. Methods A 620, 99 (2010)

62. S.A. Piriz, A.R. Piriz, N.A. Tahir, Phys. Rev. E 100, 063104 (2019)

63. A.R. Piriz, S.A. Piriz, N.A. Tahir, Phys. Rev. E 104, 035102 (2021) 
64. N.A. Tahir, A. Shutov, P. Neumayer, V. Bagnoud, A.R. Piriz, I.V. Lomonosov, S.A. Piriz, Phys. Plasmas 28, 032712 (2021)

65. B. Borm, D. Khaghani, P. Neumayer, Phys. Plasmas 26, 023109 (2019)

66. H.-S. Park, B.R. Maddox, E. Giraldez, S.P. Hatchett, L.T. Hudson, N. Izumi, M.H. Key, S. Le Pape, A.J. MacKinnon, A.C. MacPhee et al., Phys. Plasmas 15, $072705(2008)$

67. F. Albert, N. Lemos, J.L. Shaw, P.M. King, B.B. Pollock, C. Goyon, W. Shumaker, A.M. Saunders, K.A. Marsh, A. Pak et al., Nucl. Fusion 59, 032003 (2019)

68. M. J. Berger, J.H. Hubbell, S.M. Seltzer, J. Chang, J.S. Coursey, R. Sukumar, D.S. Zucker, K. Olsen (2010), XCOM: Photon Cross Section Database (version 1.5). http://physics.nist.gov/xcom. National Institute of Standards and Technology, Gaithersburg

69. H.F. Wilson, M.L. Wong, B. Militzer, Phys. Rev. Lett. 110, 151102 (2013)

70. M. Millot, F. Coppari, J.R. Rygg, A.C. Barrios, S. Hammel, D.C. Swift, J.H. Eggert, Nature 569, 251 (2019)

71. V.E. Fortov, B. Goel, C.D. Munz, A.L. Ni, A.V. Shutov, O.Y. Vorobiev, Nucl. Sci. Eng. 123, 169 (1996)

72. S.K. Godunov, V.A. Zabrodin, A.N. Kraiko, G.P. Prokopov, Nauka, Moscow (1976)

73. J.F. Ziegler, J.P. Biersack, U. Littmark, The Stopping and Ranges of Ions in Solids (Pergamon Press, New York, 1996)

74. I.V. Lomonosov, Laser Part. Beams 25, 567 (2007)

75. V.E. Fortov, I.V. Lomonosov, Phys. Usp. 57, 219 (2014)

76. SESAME: The Los Alamos Nat. Lab. EOS data, LA-UR-92-3407, Ed. P.L. Stanford and J.D. Johnson

77. A.S. Khan, S. Huang, Continuous Theory of Plasticity (Wiley, New York, 1995) 